\title{
St. Lucia-Request for Disbursement under the Rapid Credit Facility and Emergency Natural Disaster Assistance_-Staff Report, Staff Supplement, Press Release and Statement by the Executive Director for St. Lucia
}

The following documents have been released and are included in this package:

- $\quad$ The staff report for the Request for Disbursement under the Rapid Credit Facility and Emergency Natural Disaster Assistance, prepared by a staff team of the IMF, following discussions that ended on November 23, 2010 with the officials of St. Lucia on economic developments and policies. Based on information available at the time of these discussions, the staff report was completed on December 23, 2010. The views expressed in the staff report are those of the staff team and do not necessarily reflect the views of the Executive Board of the IMF.

- A staff supplement on the debt sustainability analysis.

- A press release.

- $\quad$ A statement by the Executive Director for St. Lucia

The policy of publication of staff reports and other documents allows for the deletion of market-sensitive information.

\author{
Copies of this report are available to the public from \\ International Monetary Fund • Publication Services \\ $70019^{\text {th }}$ Street, N.W. • Washington, D.C. 20431 \\ Telephone: (202) 623-7430 • Telefax: (202) 623-7201 \\ E-mail: publications@imf.org Internet: http://www.imf.org
}

\section{International Monetary Fund Washington, D.C.}


INTERNATIONAL MONETARY FUND

ST. LUCIA

\section{Request for Disbursement Under the Rapid Credit Facility and Emergency Natural Disaster Assistance}

Prepared by the Western Hemisphere Department

(in Collaboration with Other Departments)

Approved by Rodrigo Valdés (WHD) and Jan Kees Martijn (SPR)

December 23, 2010

\section{Executive Summary}

Hurricane Tomas struck St. Lucia on October 30-31, 2010, causing loss of life and significant damages to infrastructure and agriculture. Economic activity declined by 3.6 percent in 2009 and was on course for a gradual recovery in 2010. Hurricane Tomas, however, caused significant damages to the road network, the water supply, and the agriculture sector. The impact on the tourism sector, which has just started its high season, has also been quite pronounced.

St. Lucia faces difficult policy challenges in addressing the impact of the hurricane. Total damages caused by the hurricane are estimated preliminarily at US\$336 million (about 34 percent of GDP). Rehabilitation and emergency measures are currently being financed through the reallocation of the investment budget and financing already received (US\$3.2 million from the Caribbean Catastrophe Risk Insurance Facility and US $\$ 0.2$ million in emergency grants from the Caribbean Development Bank). Going forward and given the high level of the public debt, the authorities expect to finance the reconstruction through grants and external concessional financing, and have requested Fund financial assistance under the Rapid Credit Facility/Emergency Natural Disaster Assistance. The letter of intent is attached.

The authorities reiterated their commitment to achieving the medium-term debt target. While the rehabilitation and reconstruction efforts will contribute to a widening of the fiscal deficit in FY 2010/11 and FY 2011/12, the authorities plan to rein in fiscal deficits in the medium term and achieve by 2020 the regional target of a debt-to-GDP ratio of 60 percent. To this end, they remain committed to implementing revenue and expenditure measures, including a property tax in early 2011, a VAT by April 2012, and broad-based public expenditure reforms. Also, they intend to continue moving forward with structural reforms to foster private sector led growth and poverty reduction.

Access under the RCF/ENDA. On the basis of the authorities' commitment to sound macroeconomic policies, staff supports the request for access of SDR 5.36 million (US\$8.22 million), equivalent to 35 percent of quota.

Fund Relations. The Executive Board approved a request for a RAC-ESF on July 27, 2009 of an amount equivalent to SDR 6.89 million (45 percent of quota). The last Article IV consultation was concluded by the Executive Board on March 15, 2010. The staff report and summing up of the Executive Directors' discussions and policy recommendations are available at: http://www.imf.org/external/pubs/cat/longres.cfm?sk=23786.0 


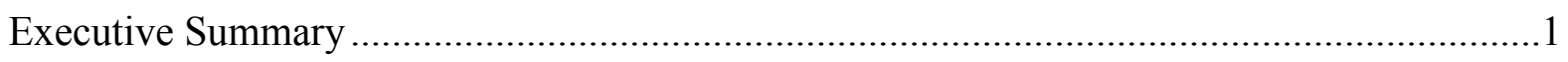

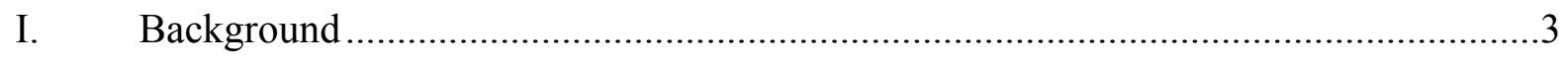

II. Economic Performance Prior to Hurricane Tomas ...................................................4

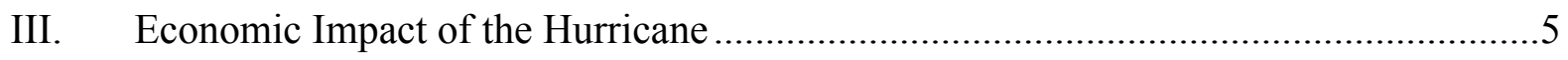

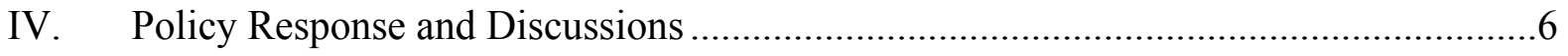

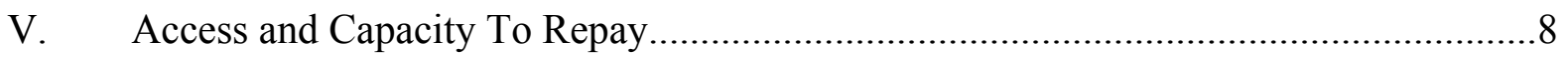

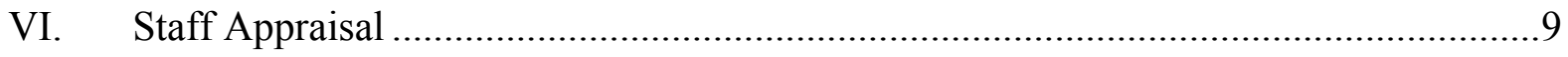

\section{Tables}

1. Selected Social and Economic Indicators, 2006-11 ............................................11

2. Operations of the Central Government, 2006-15 …........................................... 12

3. Operations of the Central Government, 2006-15 ..............................................13

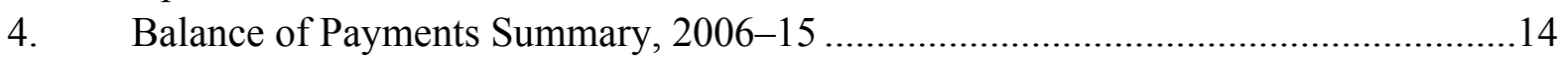

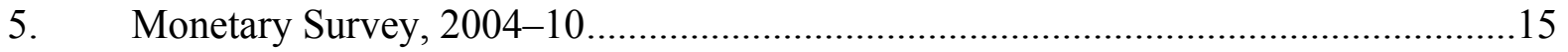

6. Indicators of External and Financial Vulnerability, 2005-10 .................................16

7. Indicators of Capacity to Repay the Fund, 2010-21 ............................................ 17

Attachment

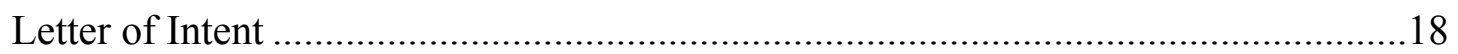




\section{BACKGROUND}

1. St. Lucia was hit by Hurricane Tomas on October 30-31, 2010, causing loss of life and considerable damage to infrastructure and agriculture. According to an UN ECLAC report, torrential rains caused landslides, resulting in human casualties and severe damages to infrastructure (the road network and water supply), and agriculture. ${ }^{1}$ Some commercial and residential buildings (including hotels) were also affected. The overall impact is estimated preliminarily at US\$336 million (about 34 percent of GDP). This includes export losses of US\$45 million.

2. St. Lucia is experiencing an urgent balance of payments need characterized by a financing gap that, if not addressed, would result in an immediate and severe economic disruption. The authorities moved quickly to provide immediate relief to those affected by the hurricane and have begun reconstruction. The public administration has responded expeditiously and effectively. So far reconstruction efforts are being financed through the reallocation of the investment budget (equivalent to about US\$28.5 million), US\$3.2 million from the Caribbean Catastrophe Risk Insurance Fund, and US\$0.2 million in emergency grants from the Caribbean Development Bank.

3. The authorities have requested a purchase equivalent to 35 percent of quota (see attached letter of intent), consisting of a disbursement of SDR 3.83 million under the Rapid Credit Facility (RCF) and a purchase equivalent to SDR 1.53 million under the Fund's Emergency Natural Disaster Assistance (ENDA). The request follows a 45 percent of quota drawing under the Exogenous Shocks Facility (RAC-ESF) ${ }^{2}$ in June 2009 aimed at mitigating the effect of the global economic and financial crisis, which underscores St. Lucia's vulnerability to sudden and exogenous shocks. Access under the RCF/ENDA would help close the financing gap in FY 2010/11. ${ }^{3}$

\footnotetext{
${ }^{1}$ ECLAC/UNDP, Saint Lucia: Macro Socio-Economic and Environmental Assessment Report: Towards Resilience Following the Passage of Hurricane Tomas, December 2010. An estimated 5,952 people, about 3.5 percent of the total population, were severely affected, including 7 deaths, 5 missing, 36 injuries and 473 in shelters.

${ }^{2}$ A number of policies envisaged under the RAC-ESF have been implemented. However, key measures (VAT and market valuation-based property tax) are still pending.

${ }^{3}$ A mission visited Castries on November 23-24 to assess the immediate balance of payments needs resulting from the impact of Hurricane Tomas, reviewed the authorities' policy measures to address the shock, and had preliminary discussion on the modalities of possible Fund financing. Final meetings were held on December 17 via VC. The staff team comprised A. Schipke (Head), K. Nassar and S. Ogawa (all WHD), and W. Samuel (Regional Resident Representative). The mission met with the Permanent Secretary of the Ministry of Finance and other senior government officials.
} 
4. The thrust of the authorities' policies in recent years has been broadly consistent with Fund advice. In the ECCU, St. Lucia is the largest economy and generally has stronger vulnerability indicators. The authorities have followed key Fund recommendations, including strengthening financial management, debt management and supervision of the nonbank financial sector, as well as pension reform. They have, however, been slow in responding to staff recommendations to implement a value-added tax and market valuation-based property $\operatorname{tax}^{4}$

\section{Economic Performance Prior to Hurricane Tomas}

5. St. Lucia's economy had begun to pick up when Hurricane Tomas hit the island. Economic activity declined by 3.6 percent in 2009 and was on a path to achieve a 1.7 percent growth in 2010, buoyed by a noticeable rebound in tourism. Stay-over tourist arrivals increased by about 15 percent through September 2010, helped by a strong marketing campaign in North America and a marked improvement in airlift (including the introduction of low cost carriers - Jet Blue and West Jet from the United States and Canada, respectively). Declines in activity in the agriculture and banking sectors, however, weighed on the recovery. Inflation remained moderate at 2.7 percent at end-September 2010.

6. Pre-hurricane fiscal imbalances were projected to remain stable in FY 2010/11. While the central government's total revenue-to-GDP ratio would have improved by 1.2 percentage points to 32.2 percent of GDP, total spending was projected to increase by about $1 \frac{1}{2}$ percentage points of GDP, mainly reflecting an increase in personal emoluments. As a result, the primary fiscal deficit (including grants) would have been largely unchanged with respect to FY 2009/10 at 0.6 percent of GDP. The overall deficit would have been 4.3 percent of GDP (0.3 percentage points higher than in FY 2009/10).

7. The external current account balance was projected to deteriorate in 2010 . The external current account deficit was expected to widen by about $2 \frac{1}{2}$ percentage points of GDP to about 17 percent of GDP, due to a decline in banana exports and an increase in construction- and fuel-related imports. Based on solid advance bookings, however, stay-over tourist arrivals were projected to remain strong. The external current account deficit was financed largely by FDI and government borrowing.

8. The adverse impact of the global economic and financial crisis put additional stress on the financial system. Despite a gradual recovery prior to Hurricane Tomas, financial soundness indicators continued to deteriorate with NPLs rising to 13.6 percent (a 5 percentage point deterioration over a 12-month period), while provisioning had fallen short

\footnotetext{
${ }^{4}$ Elections are constitutionally due by March 2012, but can be called at any time.
} 
of the increase in NPLs. Liquidity remained tight and growth of credit to the private sector also continued to be weak during 2010 (2.4 percent year-on-year). At the same time, the largest bank in St. Lucia acquired a majority stake in the privatization of the National Bank of St. Vincent and the Grenadines.

\section{ECONOMIC IMPACT OF THE HURRICANE}

\section{Preliminary estimates suggest that growth could be constrained to 0.5 percent}

in 2010. Damages to

the road network, utilities and agricultureespecially bananaswere severe, accounting for the bulk of the slowdown with negative spillovers

St. Lucia: Output and Prices, 2010-11 (Annual percentage change, unless otherwise specified)

\begin{tabular}{|c|c|c|c|c|c|c|}
\hline & \multirow{2}{*}{2009} & \multicolumn{3}{|c|}{2010} & \multicolumn{2}{|c|}{2011} \\
\hline & & Art. IV & $\begin{array}{c}\text { Rev. proj. } \\
1 /\end{array}$ & $\frac{\text { RCF/ENDA }}{2 /}$ & Art. IV & RCF/ENDA \\
\hline $\begin{array}{l}\text { Real GDP at factor cost } \\
\text { Of which: }\end{array}$ & -3.6 & 1.1 & 1.7 & 0.5 & 2.3 & 4.1 \\
\hline Agriculture & -3.4 & 2.5 & -8.6 & -17.7 & 10.0 & -11.2 \\
\hline Housing & 0.0 & 1.0 & 1.0 & -0.9 & 2.0 & -1.8 \\
\hline Tourism & -7.0 & 3.0 & 15.5 & 8.0 & 3.0 & 4.8 \\
\hline Utilities & 4.0 & 1.0 & 4.5 & 2.3 & 0.5 & 6.8 \\
\hline GDP at current market prices & -3.8 & 2.8 & 4.0 & 2.8 & 4.8 & 5.8 \\
\hline GDP deflator at factor cost & -0.2 & 1.7 & 2.2 & 2.2 & 2.5 & 1.6 \\
\hline Consumer prices (end of period) & 1.0 & 1.9 & 1.3 & 1.4 & 2.1 & 2.1 \\
\hline
\end{tabular}

Sources: Ministry of Finance; and Fund staff estimates and projections.

$1 /$ Revised based on the outturn through end-September, 2010.

2/ Reflects the impact of Hurricane Tomas on agriculture, housing, tourism and utilities.

to other sectors.

Although temporary, the impact on the tourism sector was also quite pronounced. The economy is, however, expected to grow by about 4.1 percent in 2011 , about 1.8 percentage points higher than the pre-hurricane forecast, as rehabilitation and reconstruction efforts commence in earnest.

\section{The fiscal impact of the hurricane will be felt in both FY 2010/11 and}

FY 2011/12. In

FY 2010/11, the

revenue impact is estimated at about half a percentage point of GDP. The additional spending needed to provide assistance to those who have been adversely impacted and undertake emergency

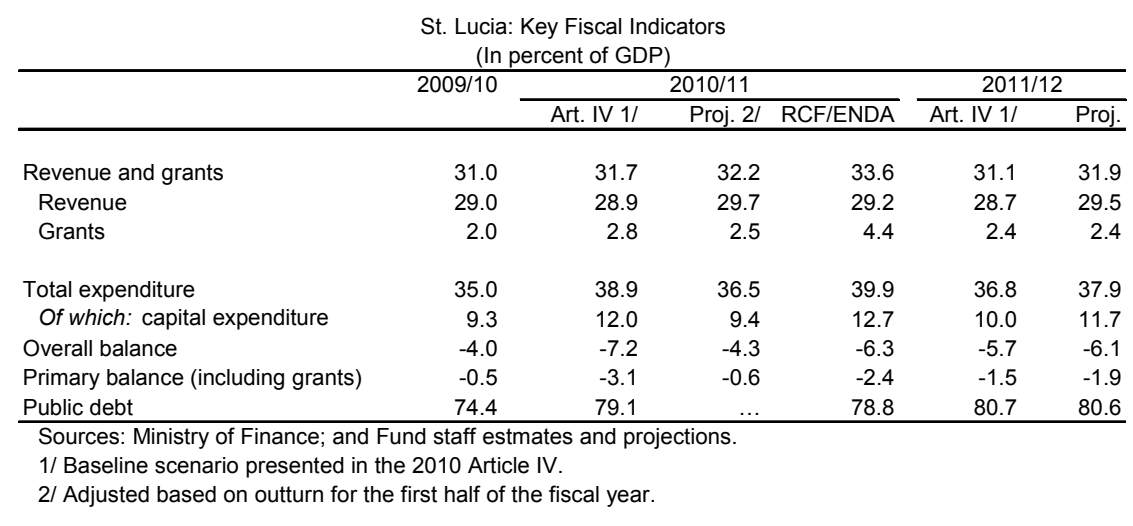
rehabilitation of infrastructure (the road network and water supply) is estimated at 3 percent of GDP. ${ }^{5}$ The increased expenditure will raise the overall fiscal deficit to 6.3 percent of GDP

\footnotetext{
${ }^{5}$ The final figure is not yet known, as the authorities have not yet tabled the supplementary budget in parliament.
} 
in FY 2010/11 and 6.1 percent of GDP in FY2011/12 raising the public debt level to about 81 percent of GDP in FY2011/12.

\section{The balance of payments impact of the hurricane will be significant in}

\section{calendar year}

2011. Reflecting

higher

reconstruction-

related imports

and the damage to

the banana crop,

the external

current account
St. Lucia: Key External Sector Indicators (In percent of GDP; unless otherwise indicated)

\begin{tabular}{lrrrrrr}
\hline & 2009 & \multicolumn{2}{c}{2010} & & \multicolumn{2}{c}{2011} \\
& Prel. & Art. IV 1/ & RCF/ENDA & Art. IV 1/ & RCF/ENDA \\
\hline External current account balance & -14.4 & -21.2 & -16.7 & & -22.1 & -25.4 \\
Exports of goods and nonfactor services & 56.7 & 51.0 & 58.6 & & 53.1 & 56.3 \\
Imports of goods and nonfactor services & 66.9 & 65.8 & 70.7 & & 68.9 & 77.1 \\
Overall balance & 3.1 & 0.3 & -0.3 & & 0.6 & -3.7 \\
Identified hurricane-related financing & 0.0 & 0.0 & 0.0 & & 0.0 & 3.8 \\
RCF/ENDA & & & & & \\
$\quad$ in percent of identified financing & & & & & & \\
\hline Sources: ECCB, and Fund staff estimates. & & & & & & \\
1/ Baseline scenario presented in 2010 Article IV. & & & & & \\
\hline
\end{tabular}

deficit is set to widen in 2011. The rise in imports also reflects the recent increase in the international prices of energy and food. The current account deficit is projected to deteriorate by about 9 percentage points of GDP, contributing to an overall balance of payments deficit of 3.7 percent of GDP.

\section{Despite increased stress related to the global financial crisis, the banking} sector appears to be in a position to weather the effects of the hurricane. Despite adverse developments, capital adequacy ratios remained high at 19.9 percent as of September 2010, well above the prudential requirements ( 8 percent). However, credit unions and cooperatives in the banana sector should brace for an increase in NPLs. The authorities are monitoring developments closely and intend to strengthen nonbank financial sector legislation and supervision (see LOI paragraph 4).

\section{Policy Response And Discussions}

\section{St. Lucia faces difficult policy challenges in addressing the impact of the}

hurricane. First, as a member of the Eastern Caribbean Currency Union (ECCU) and with a quasi currency board, the country is constrained from using monetary policy. Second, the high level of public debt leaves limited fiscal room to address the impact of the hurricane. Third, although St. Lucia's public administration has been effective in dealing with the crisis, there are capacity limitations in scaling up public investment. Given these constraints, the authorities intend to limit the financing of the rehabilitation and reconstruction efforts primarily to grants and external concessional financing, focusing on emergency support for those affected the most, the road network, and water. 


\section{Despite a temporary deterioration in the fiscal position, the authorities} reiterated their commitment to achieving the medium-term debt target. Total capital expenditure will be curtailed to about

12.7 percent of GDP in

FY2010/11 and

11.7 percent in FY2011/12, in line with available concessional funding and additional revenue measures

St. Lucia: Identified Financing for Hurricane-Related Spending (in millions of EC dollars)

\begin{tabular}{lrrr}
\hline & FY2010/11 & FY2011/12 & $\begin{array}{r}\text { Total equival. to } \\
\text { calendar year 2011 }\end{array}$ \\
\hline CDB & 2.0 & 59.0 & 61.0 \\
IMF (RCF/ENDA) & 22.1 & & 22.1 \\
CARICOM Development Fund & 4.1 & & 4.1 \\
World Bank (US\$5 million) & 13.5 & & 13.5 \\
EU & & 7.0 & 7.0 \\
Total & 41.7 & $\mathbf{6 6 . 0}$ & $\mathbf{1 0 7 . 7}$ \\
\hline
\end{tabular}

Sources: Ministry of Finance.

(as described below). As

detailed in the attached letter of intent, the impact of the reconstruction efforts would temporarily lead to a primary fiscal deficit of 2.4 percent of GDP in FY 2010/11 and 1.9 in FY 2011/12. The authorities have identified sources of financing in the form of additional grants and concessional loans to close the gaps and intend to resort to bridge financing (via the Regional Government Securities Market, a bond issuance outside of the region, or commercial bank borrowing), if the disbursement of the identified financing were delayed. While the widening of the fiscal deficit would increase the public debt-to-GDP ratio, the authorities reaffirmed their commitment to reaching the targeted debt-to-GDP ratio of 60 percent by 2020 .

\section{To reach the debt target, the authorities plan to implement both revenue and} expenditure measures to achieve a primary surplus of 2.9 percent of GDP in the medium term, compared with 1.6 percent of GDP recommended in the Article IV. On the revenue side, Cabinet has recently approved legislation to implement a market valuation-based property tax and plan to present the bill to Parliament in early 2011, which would yield an additional 0.3 percent of GDP. In addition, the authorities strongly confirmed their commitment to introduce a VAT by April 2012 and have already started the consultative process with the

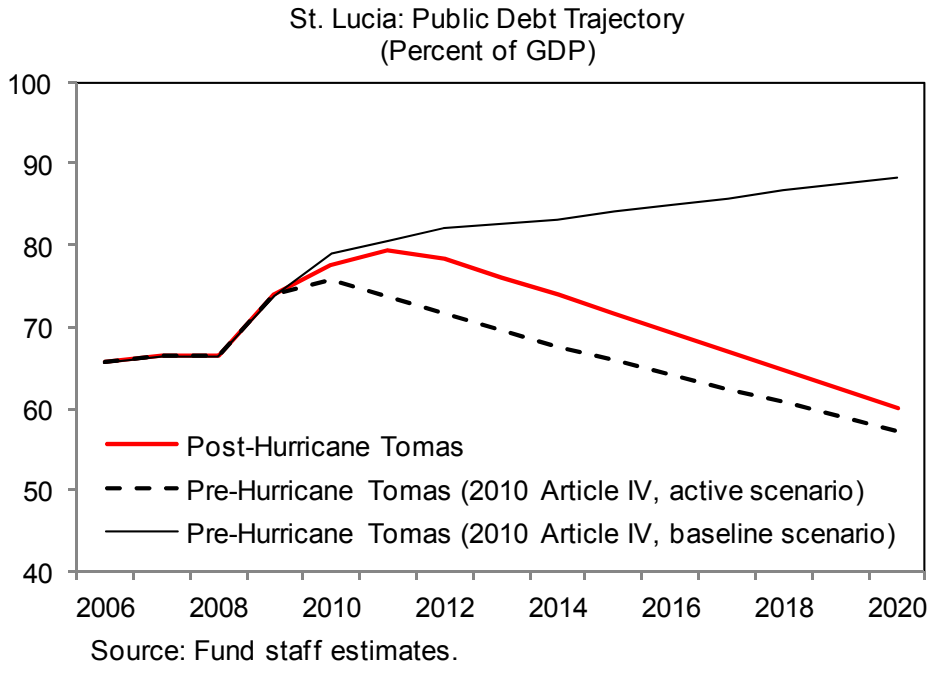
private sector and other key stakeholders. On the expenditure front, the authorities intend to freeze the civil service wage bill at 11 percent of GDP within the context of broader civil service reforms aimed at increasing productivity and efficiency. Also, they will implement a formal public sector 
investment program, underpinned by a medium-term expenditure framework, and are seeking technical assistance from CARTAC.

\section{The authorities intend to press ahead with structural reforms aimed at} bolstering economic growth and reducing poverty. They are determined to continue enhancing the investment climate for private sector development, including establishing a one-stop shop for investors. Also, to promote a better allocation of capital and strengthen oversight and resilience of the financial sector, the authorities will establish the Single Regulatory Unit and seek passage of credit union legislation. ${ }^{6}$ Both pieces of legislation have been approved by Cabinet

\section{ACCESS AND CAPACITY To RePay}

\section{The authorities have requested fund financing in an amount equivalent to} 35 percent of quota in total, SDR 5.36 million (25 percent of quota) under the Rapid Credit Facility, supplemented by the Emergency Natural Disaster Assistance. ${ }^{7}$ At this juncture, the authorities do not intend to request an ECF. Furthermore, given the urgent need for rapid support, there is not enough time to engage in prolonged negotiations on a Fund arrangement. The purchase - which represents approximately 0.8 percent of St. Lucia's GDP in direct budgetary support — would help meet the immediate foreign exchange needs stemming from the hurricane. More importantly, while the purchase accounts for about 20 percent of the financing gap in 2011, it is expected to play a catalytic role in mobilizing financial support from other international/regional financial institutions. Staff will raise the possibility of a formal Fund arrangement in the context of the forthcoming Article IV consultation, tentatively scheduled for end March/beginning of April, to support the authorities' policies for addressing the medium-term fiscal and debt challenges.

\section{It is expected that St. Lucia will be able to discharge its obligations to the Fund}

in a timely manner. St. Lucia's outstanding loans to the Fund are 45 percent of quota, which would increase to 80 percent. While St. Lucia remains vulnerable to implementation risks and future exogenous shocks (including natural disasters), staff judges that the risks are mitigated by the countries' exemplary debt servicing record and proven commitment to prudent fiscal policies prior to the global financial and economic crisis. The authorities are committed to reining in expenditures and following through on revenue reforms that will put the debt on a firmly downward trajectory beginning in FY 2012/13.

\footnotetext{
${ }^{6}$ Both pieces of legislation have already been approved by Cabinet.

${ }^{7}$ Regarding the proposed access level, debt vulnerabilities and repeated use of shocks instruments argue for keeping some room under the annual and cumulative ceilings. The authorities' policies are sufficiently strong to address the shock. The blending proposal is in line with the presumption that for PRGT-eligible countries with a per-capita GNI above the IDA operational cutoff, overall access is provided under blended PRGT and GRA instruments (taking into account the minimum access level under the PRGT at 25 percent of quota).
} 
19. Under the Fund's safeguards assessment policy, the Eastern Caribbean Central Bank (ECCB) is subject to a full safeguards assessment under a four year cycle. The most recent assessment was completed in July 2007, and concluded that the ECCB continues to have appropriate control mechanisms in place, which have strengthened since the first safeguards assessment completed in 2003. ECCB management places emphasis on good governance and sound controls, and has enhanced the bank's transparency and accountability since the last assessment, including through the publication of financial statements that comply with International Financial Reporting Standards. The next assessment will take place in 2011.

\section{Staff APPRAisal}

\section{St. Lucia faces significant policy challenges in the aftermath of Hurricane}

Tomas. Staff estimates that economic activity, which was gradually recovering, will remain subdued in 2010, on account of extensive damages caused by the hurricane. While the medium-term outlook remains positive, it would take considerable efforts and resources to put the economy back on its pre-hurricane trend growth rate. Given the increased spending on reconstruction, the economy should enjoy a solid 4.1 percent growth in 2011.

\section{The policies outlined in the letter of intent tackle urgent rebuilding needs and} appropriately aim to maintain macroeconomic stability. In view of the high public debtto-GDP ratio, staff supports the authorities' strategy to limit the financing of the rehabilitation and reconstruction efforts to additional grants and external concessional financing. In this context, access under the RCF/ENDA and financial support from other international/regional financial institutions and donor countries will be key to maintaining macroeconomic stability. Also, the authorities' plans to implement structural reforms that aim to foster private sector-led growth, including improving economic efficiency and competitiveness, are critical.

\section{The authorities are rightly focused on achieving medium-term debt}

sustainability. In the short term, the reconstruction needs are set to put upward pressure on the fiscal position. In this context, the governments' public commitment - as reflected in the letter of intent - to implement tax and expenditure reforms, including a market-based property tax in early 2011 and the introduction of a VAT by April 2012, is critical. These reforms, coupled with implementation of a formal public sector investment program and a medium-term expenditure framework to raise spending efficiency, should secure fiscal consolidation and put public debt on a firmly downward trajectory in the medium term.

\section{Staff supports the authorities' request for a disbursement under the Fund's}

RCF/ENDA. Staff's support is based on the extent of the damage caused by Hurricane Tomas, the associated urgent balance of payments need, and the authorities' commitments to limiting the temporary increase in capital spending to additional grants and concessional financing, while committing to achieving the debt-to-GDP target of 60 percent by 2020 by 
implementing revenue and expenditure measures as outlined in the authorities' letter of intent. There are, however, downside risks such as the country's high public debt, inherent vulnerability to exogenous shocks, and election cycle related policy implementation delays, all of which pose some risks to the Fund's resources. These risks are mitigated by the authorities' track record of sound fiscal policies, implementation of Fund recommendations, resolve to achieving fiscal consolidation in the medium term and fostering private sector-led growth, and commitment to a continued close dialogue with the Fund. 
Table 1. St. Lucia: Selected Social and Economic Indicators, 2006-11

\begin{tabular}{|c|c|c|c|c|c|c|}
\hline I. S & Ind Demog & Indica & & & & \\
\hline $\begin{array}{l}\text { Area (sq. km) } \\
\text { Population } \\
\text { Total (2007) } \\
\text { Rate of growth (percent per year) } \\
\text { Population density (per sq. km., 2007) } \\
\text { Net migration rate (per thousand, 2002) } \\
\text { Adult illiteracy rate (percent, 2004) }\end{array}$ & $\begin{array}{r}171,226 \\
1.4 \\
317.6 \\
-9.8 \\
5.2\end{array}$ & & $\begin{array}{l}\text { pectanc } \\
\text { nortality } \\
\text { Develo } \\
\text { out of } \\
\text { Domest } \\
\text { ons of } \\
\text { ons of } \\
\text { per cap }\end{array}$ & $\begin{array}{l}\text { th (year } \\
\text { nousand } \\
\text { Index ( } \\
\text { untries) } \\
\text { uct (200 } \\
\text { ars) } \\
\text { ars) }\end{array}$ & $\begin{array}{l}\text { 6) } \\
\text { inths, } 2 C \\
\text { Inking ( }\end{array}$ & $\begin{array}{r}996 \\
2,690 \\
5,819\end{array}$ \\
\hline II. Econo & nd Financi & cators, & & & & \\
\hline & 2006 & 2007 & 2008 & 2009 & $\begin{array}{r}\text { Est. } \\
2010\end{array}$ & $\begin{array}{l}\text { Proj. } \\
2011\end{array}$ \\
\hline & & al perce & change, & otherw & ecified) & \\
\hline $\begin{array}{l}\text { Output and prices } \\
\text { Real GDP at factor cost } \\
\text { GDP at current market prices } \\
\text { GDP deflator at factor cost } \\
\text { Consumer prices (end of period) } \\
\text { Consumer prices (period average) } \\
\text { Banana export receipts } \\
\text { Unemployment rate (in percent) }\end{array}$ & $\begin{array}{r}4.8 \\
6.1 \\
2.5 \\
1.4 \\
4.1 \\
15.9 \\
16.6\end{array}$ & $\begin{array}{r}1.5 \\
2.9 \\
0.7 \\
6.6 \\
2.2 \\
-9.2 \\
13.9\end{array}$ & $\begin{array}{r}0.7 \\
3.8 \\
3.1 \\
3.8 \\
7.2 \\
35.0 \\
16.8\end{array}$ & $\begin{array}{r}-3.6 \\
-3.8 \\
-0.2 \\
1.0 \\
1.0 \\
-0.8 \\
\quad \ldots\end{array}$ & $\begin{array}{r}0.5 \\
2.8 \\
2.2 \\
1.4 \\
2.2 \\
-29.7 \\
\quad \ldots\end{array}$ & $\begin{array}{r}4.1 \\
5.8 \\
1.6 \\
2.1 \\
1.6 \\
-20.4 \\
\quad \ldots\end{array}$ \\
\hline $\begin{array}{l}\text { External sector } \\
\text { Exports, f.o.b. } \\
\text { Imports, f.o.b. } \\
\text { Travel receipts } \\
\text { Terms of trade (- = deterioration) } \\
\text { Real effective exchange rate (end of } \\
\quad \text { period, - = depreciation ) }\end{array}$ & $\begin{array}{r}32.2 \\
24.6 \\
-20.0 \\
-12.7 \\
0.3\end{array}$ & $\begin{array}{r}-5.0 \\
4.0 \\
11.6 \\
-11.2 \\
-3.8\end{array}$ & $\begin{array}{l}48.6 \\
-1.3 \\
-2.1 \\
-5.5 \\
0.5\end{array}$ & $\begin{array}{r}15.4 \\
-15.6 \\
-4.8 \\
14.9 \\
3.2\end{array}$ & $\begin{array}{l}-8.7 \\
11.9 \\
16.2 \\
-6.5\end{array}$ & $\begin{array}{r}-12.5 \\
19.8 \\
7.5 \\
-0.2\end{array}$ \\
\hline $\begin{array}{l}\text { Money and credit } 1 / \\
\text { Net foreign assets } \\
\text { Net domestic assets } \\
\text { Of which } \\
\text { Credit to private sector }\end{array}$ & $\begin{array}{r}1.0 \\
19.1\end{array}$ & $\begin{array}{l}-8.0 \\
14.2 \\
34.2\end{array}$ & $\begin{array}{r}-22.7 \\
35.7 \\
\\
27.0\end{array}$ & $\begin{array}{l}1.2 \\
0.3 \\
2.6\end{array}$ & $\begin{array}{r}-1.7 \\
4.5 \\
1.9\end{array}$ & $\begin{array}{r}-2.0 \\
6.8\end{array}$ \\
\hline & & perce & DP, unle & erwise & & \\
\hline $\begin{array}{l}\text { Central government } 2 \text { I } \\
\text { Total revenue and grants } \\
\text { Total expenditure and net lending } \\
\text { Current expenditure } \\
\text { Of which }\end{array}$ & $\begin{array}{l}26.7 \\
32.8 \\
21.6\end{array}$ & $\begin{array}{l}28.4 \\
29.4 \\
22.4\end{array}$ & $\begin{array}{l}30.6 \\
31.7 \\
24.3\end{array}$ & $\begin{array}{l}31.0 \\
35.0 \\
25.7\end{array}$ & $\begin{array}{l}33.6 \\
39.9 \\
27.3\end{array}$ & $\begin{array}{l}31.9 \\
37.9 \\
26.2\end{array}$ \\
\hline $\begin{array}{l}\text { Wages and salaries } \\
\text { Interest } \\
\text { Capital expenditure } \\
\text { Overall balance (cash basis) } \\
\text { Of which }\end{array}$ & $\begin{array}{r}9.9 \\
3.3 \\
11.2 \\
-6.1\end{array}$ & $\begin{array}{r}10.1 \\
3.6 \\
7.0 \\
-1.0\end{array}$ & $\begin{array}{r}11.2 \\
3.4 \\
7.5 \\
-1.1\end{array}$ & $\begin{array}{r}12.1 \\
3.4 \\
9.3 \\
-4.0\end{array}$ & $\begin{array}{r}12.6 \\
3.9 \\
12.7 \\
-6.3\end{array}$ & $\begin{array}{r}11.8 \\
4.2 \\
11.7 \\
-6.1\end{array}$ \\
\hline $\begin{array}{l}\text { Current balance (savings) } \\
\text { Primary balance (after grants) } \\
\text { Central government debt } \\
\text { Debt service in percent of current revenues } 3 /\end{array}$ & $\begin{array}{r}4.8 \\
-2.8 \\
63.5 \\
35.7\end{array}$ & $\begin{array}{r}5.8 \\
2.6 \\
64.5 \\
39.9\end{array}$ & $\begin{array}{r}5.3 \\
2.3 \\
64.8 \\
24.6\end{array}$ & $\begin{array}{r}3.3 \\
-0.5 \\
72.2 \\
53.6\end{array}$ & $\begin{array}{r}1.9 \\
-2.4 \\
76.1 \\
53.4\end{array}$ & $\begin{array}{r}3.2 \\
-1.9 \\
78.0 \\
35.2\end{array}$ \\
\hline $\begin{array}{l}\text { External sector } \\
\text { External current account } \\
\text { Of which }\end{array}$ & -30.2 & -31.3 & -27.8 & -14.4 & -16.7 & -25.4 \\
\hline $\begin{array}{l}\text { Exports of goods and services } \\
\text { Imports of goods and services } \\
\text { Stayover arrivals (percentage change) } \\
\text { Foreign direct investment (FDI) }\end{array}$ & $\begin{array}{l}48.4 \\
73.9 \\
-4.9 \\
25.1\end{array}$ & $\begin{array}{l}50.4 \\
76.0 \\
-5.0 \\
28.3\end{array}$ & $\begin{array}{r}53.1 \\
75.3 \\
2.9 \\
15.3\end{array}$ & $\begin{array}{l}56.7 \\
66.9 \\
-8.0 \\
15.3\end{array}$ & $\begin{array}{l}58.6 \\
70.7 \\
14.3 \\
13.1\end{array}$ & $\begin{array}{r}56.3 \\
77.1 \\
5.4 \\
13.3\end{array}$ \\
\hline $\begin{array}{l}\text { Public sector external debt (end of period) } \\
\text { External public debt service } 4 /\end{array}$ & 44.8 & 41.8 & 36.5 & 39.7 & 39.5 & 40.0 \\
\hline $\begin{array}{l}\text { In percent of exports of goods and services } \\
\text { In percent of central government revenue before grants }\end{array}$ & $\begin{array}{l}11.2 \\
22.1\end{array}$ & $\begin{array}{l}17.0 \\
32.4\end{array}$ & $\begin{array}{l}11.4 \\
22.1\end{array}$ & $\begin{array}{l}11.2 \\
23.3\end{array}$ & $\begin{array}{r}9.1 \\
19.1\end{array}$ & $\begin{array}{r}9.5 \\
18.9\end{array}$ \\
\hline $\begin{array}{l}\text { Memorandum items: } \\
\text { Gross public sector debt } 5 / 6 / 7 / \\
\text { Nominal GDP at market prices (in millions of EC dollars) } \\
\text { Nominal GDP at factor cost (in millions of EC dollars) } \\
\text { Share of ECCU stayover visitors }\end{array}$ & $\begin{array}{r}66.1 \\
2,520 \\
2,066 \\
27.9\end{array}$ & $\begin{array}{r}67.1 \\
2,592 \\
2,111 \\
27.0\end{array}$ & $\begin{array}{r}65.8 \\
2,690 \\
2,171 \\
27.9\end{array}$ & $\begin{array}{r}74.4 \\
2,589 \\
2,089 \\
29.7\end{array}$ & $\begin{array}{r}78.8 \\
2,660 \\
2,152\end{array}$ & $\begin{array}{r}80.6 \\
2,814 \\
2,276\end{array}$ \\
\hline
\end{tabular}

Sources: St. Lucia authorities; ECCB; and Fund staff estimates and projections.

$1 /$ Changes in relation to liabilities to private sector at beginning of period.

2/ Data are for fiscal years beginning April 1.

$3 /$ Comprises domestic and external interest and amortization.

4/ Comprises external interest and amortization.

5/ Total public (including nonguaranteed) debt in percent of GDP.

$6 /$ Includes liabilities to the National Insurance Corporation (NIC).

7/ Includes liabilities related to the construction and financing of public proiects by the private sector. 
Table 2. St. Lucia: Operations of the Central Government, 2006-15 1/ (In millions of EC dollars)

\begin{tabular}{|c|c|c|c|c|c|c|c|c|c|c|}
\hline & \multirow[b]{2}{*}{2006} & \multirow[b]{2}{*}{2007} & \multirow[b]{2}{*}{2008} & \multirow[b]{2}{*}{2009} & \multirow{2}{*}{$\begin{array}{r}\text { Est. } \\
2010\end{array}$} & \multicolumn{5}{|c|}{ Projections } \\
\hline & & & & & & 2011 & 2012 & 2013 & 2014 & 2015 \\
\hline Total revenue and grants & 677.5 & 742.8 & 816.5 & 809.1 & 907.7 & 909.9 & 945.0 & $1,025.7$ & $1,079.2$ & $1,135.5$ \\
\hline Current revenue & 670.2 & 738.6 & 789.3 & 756.3 & 787.8 & 841.4 & 918.5 & 997.8 & $1,049.8$ & $1,104.7$ \\
\hline Tax revenue & 619.8 & 684.6 & 736.2 & 702.8 & 746.8 & 798.6 & 873.3 & 950.3 & 999.8 & $1,052.0$ \\
\hline Nontrade tax & 381.5 & 438.6 & 492.7 & 471.5 & 481.2 & 517.7 & 576.8 & 638.4 & 671.6 & 706.7 \\
\hline Trade tax & 238.4 & 246.0 & 243.5 & 231.3 & 265.6 & 280.8 & 296.5 & 311.9 & 328.2 & 345.3 \\
\hline Nontax revenue & 50.4 & 54.1 & 53.1 & 53.6 & 41.0 & 42.8 & 45.2 & 47.5 & 50.0 & 52.6 \\
\hline Capital revenue & 0.7 & 0.0 & 6.7 & 0.0 & 0.2 & 0.0 & 0.0 & 0.0 & 0.0 & 0.0 \\
\hline Grants & 6.6 & 4.1 & 20.5 & 52.7 & 119.7 & 68.5 & 26.5 & 27.9 & 29.3 & 30.9 \\
\hline Total expenditure and net lending & 832.6 & 768.5 & 845.9 & 912.7 & $1,077.8$ & $1,082.9$ & $1,034.8$ & $1,078.2$ & $1,126.6$ & $1,183.0$ \\
\hline Current expenditure & 548.5 & 586.5 & 647.0 & 671.1 & 735.5 & 749.0 & 778.6 & 805.6 & 828.2 & 868.9 \\
\hline Wages and salaries & 250.9 & 265.2 & 298.9 & 316.5 & 340.2 & 337.3 & 342.3 & 348.7 & 366.8 & 386.0 \\
\hline NIC contributions and retirement & 43.3 & 49.2 & 51.4 & 57.4 & 64.9 & 57.1 & 60.3 & 63.4 & 66.7 & 70.2 \\
\hline Goods and services & 101.5 & 114.1 & 131.7 & 130.5 & 142.5 & 148.8 & 152.7 & 158.5 & 150.1 & 157.9 \\
\hline Transfers & 68.5 & 65.1 & 74.2 & 77.1 & 82.5 & 85.7 & 91.2 & 95.9 & 100.9 & 106.2 \\
\hline Interest payments & 84.3 & 92.9 & 90.8 & 89.7 & 105.3 & 120.2 & 132.1 & 139.1 & 143.6 & 148.6 \\
\hline Domestic & 27.7 & 30.7 & 44.1 & 46.2 & 50.8 & 64.4 & 72.4 & 77.0 & 80.1 & 83.8 \\
\hline External & 56.6 & 62.3 & 46.8 & 43.5 & 54.5 & 55.7 & 59.7 & 62.1 & 63.5 & 64.7 \\
\hline Capital expenditure and net lending & 284.1 & 181.9 & 198.9 & 241.6 & 342.3 & 333.9 & 256.1 & 272.6 & 298.5 & 314.1 \\
\hline Primary balance & -70.8 & 67.2 & 61.4 & -13.9 & -64.9 & -52.8 & 42.3 & 86.6 & 96.1 & 101.2 \\
\hline (excluding grants) & -77.4 & 63.1 & 40.9 & -66.6 & -184.6 & -121.4 & 15.8 & 58.7 & 66.8 & 70.3 \\
\hline Current balance & 121.7 & 152.1 & 142.3 & 85.2 & 52.3 & 92.4 & 139.9 & 192.2 & 221.7 & 235.8 \\
\hline Overall balance (excluding grants) & -161.7 & -29.8 & -49.9 & -156.4 & -289.8 & -241.5 & -116.3 & -80.4 & -76.8 & -78.3 \\
\hline Overall balance (including grants) & -155.1 & -25.7 & -29.4 & -103.6 & -170.2 & -173.0 & -89.8 & -52.5 & -47.5 & -47.4 \\
\hline Financing & 166.3 & 85.4 & 85.8 & 103.6 & 170.2 & 173.0 & 89.8 & 52.5 & 47.5 & 47.4 \\
\hline External (net) & 58.5 & -22.6 & 1.6 & 35.7 & 23.4 & 74.7 & 44.9 & 26.3 & 23.7 & 23.7 \\
\hline Loans & 58.5 & -22.6 & 0.6 & 35.7 & 23.4 & 74.7 & 44.9 & 26.3 & 23.7 & 23.7 \\
\hline Drawings & 99.6 & 122.5 & 65.2 & 142.4 & 111.3 & 170.0 & 154.6 & 111.8 & 148.0 & 151.0 \\
\hline \multicolumn{11}{|l|}{ Of which: } \\
\hline Identified hurricane financing & $\ldots$ & $\ldots$ & $\ldots$ & $\ldots$ & 41.7 & 66.0 & $\ldots$ & $\ldots$ & $\ldots$ & $\ldots$ \\
\hline Amortization & 41.1 & 145.1 & 64.6 & 106.7 & 87.9 & 95.3 & 109.7 & 85.6 & 124.3 & 127.3 \\
\hline Domestic financing & 107.7 & 108.0 & 84.2 & 68.0 & 146.7 & 98.3 & 44.9 & 26.3 & 23.7 & 23.7 \\
\hline ECCB (net) & 1.1 & -4.0 & -2.3 & 0.0 & 0.0 & 0.0 & 0.0 & 0.0 & 0.0 & 0.0 \\
\hline Commercial banks (net) & 67.0 & 71.1 & 2.6 & -67.5 & 15.0 & 40.0 & 11.2 & 6.6 & 5.9 & 5.9 \\
\hline Other domestic financing & 39.7 & 40.9 & 83.9 & 135.5 & 131.7 & 58.3 & 33.7 & 19.7 & 17.8 & 17.8 \\
\hline Statistical discrepancy & -11.2 & -59.7 & 104.5 & 0.0 & 0.0 & 0.0 & 0.0 & 0.0 & 0.0 & 0.0 \\
\hline
\end{tabular}

Sources: St. Lucia authorities; and Fund staff estimates and projections.

1/ Data are for fiscal years beginning April 1. 
Table 3. St. Lucia: Operations of the Central Government, 2006-15 1/ (In percent of GDP)

\begin{tabular}{|c|c|c|c|c|c|c|c|c|c|c|}
\hline & \multirow[b]{2}{*}{2006} & \multirow[b]{2}{*}{2007} & \multirow[b]{2}{*}{2008} & \multirow[b]{2}{*}{2009} & \multirow{2}{*}{$\begin{array}{r}\text { Est. } \\
2010\end{array}$} & \multicolumn{5}{|c|}{ Projections } \\
\hline & & & & & & 2011 & 2012 & 2013 & 2014 & 2015 \\
\hline Total revenue and grants & 26.7 & 28.4 & 30.6 & 31.0 & 33.6 & 31.9 & 31.4 & 32.4 & 32.4 & 32.4 \\
\hline Current revenue & 26.4 & 28.2 & 29.6 & 29.0 & 29.2 & 29.5 & 30.5 & 31.5 & 31.5 & 31.5 \\
\hline Tax revenue & 24.4 & 26.2 & 27.6 & 27.0 & 27.7 & 28.0 & 29.0 & 30.0 & 30.0 & 30.0 \\
\hline Nontrade tax & 15.0 & 16.8 & 18.5 & 18.1 & 17.8 & 18.1 & 19.1 & 20.1 & 20.1 & 20.1 \\
\hline Trade tax & 9.4 & 9.4 & 9.1 & 8.9 & 9.8 & 9.8 & 9.8 & 9.8 & 9.8 & 9.8 \\
\hline Nontax revenue & 2.0 & 2.1 & 2.0 & 2.1 & 1.5 & 1.5 & 1.5 & 1.5 & 1.5 & 1.5 \\
\hline Capital revenue & 0.0 & 0.0 & 0.3 & 0.0 & 0.0 & 0.0 & 0.0 & 0.0 & 0.0 & 0.0 \\
\hline Grants & 0.3 & 0.2 & 0.8 & 2.0 & 4.4 & 2.4 & 0.9 & 0.9 & 0.9 & 0.9 \\
\hline Total expenditure and net lending & 32.8 & 29.4 & 31.7 & 35.0 & 39.9 & 37.9 & 34.3 & 34.0 & 33.8 & 33.7 \\
\hline Current expenditure & 21.6 & 22.4 & 24.3 & 25.7 & 27.3 & 26.2 & 25.8 & 25.4 & 24.8 & 24.8 \\
\hline Wages and salaries & 9.9 & 10.1 & 11.2 & 12.1 & 12.6 & 11.8 & 11.4 & 11.0 & 11.0 & 11.0 \\
\hline $\mathrm{NIC}$ contributions and retirement & 1.7 & 1.9 & 1.9 & 2.2 & 2.4 & 2.0 & 2.0 & 2.0 & 2.0 & 2.0 \\
\hline Goods and services & 4.0 & 4.4 & 4.9 & 5.0 & 5.3 & 5.2 & 5.1 & 5.0 & 4.5 & 4.5 \\
\hline Transfers & 2.7 & 2.5 & 2.8 & 3.0 & 3.1 & 3.0 & 3.0 & 3.0 & 3.0 & 3.0 \\
\hline Interest payments & 3.3 & 3.6 & 3.4 & 3.4 & 3.9 & 4.2 & 4.4 & 4.4 & 4.3 & 4.2 \\
\hline Domestic & 1.1 & 1.2 & 1.7 & 1.8 & 1.9 & 2.3 & 2.4 & 2.4 & 2.4 & 2.4 \\
\hline External & 2.2 & 2.4 & 1.8 & 1.7 & 2.0 & 2.0 & 2.0 & 2.0 & 1.9 & 1.8 \\
\hline Capital expenditure and net lending & 11.2 & 7.0 & 7.5 & 9.3 & 12.7 & 11.7 & 8.5 & 8.6 & 9.0 & 9.0 \\
\hline $\begin{array}{l}\text { Primary balance } \\
\text { (excluding grants) }\end{array}$ & $\begin{array}{l}-2.8 \\
-3.1\end{array}$ & $\begin{array}{l}2.6 \\
2.4\end{array}$ & $\begin{array}{l}2.3 \\
1.5\end{array}$ & $\begin{array}{l}-0.5 \\
-2.6\end{array}$ & $\begin{array}{l}-2.4 \\
-6.8\end{array}$ & $\begin{array}{l}-1.9 \\
-4.3\end{array}$ & $\begin{array}{l}1.4 \\
0.5\end{array}$ & $\begin{array}{l}2.7 \\
1.9\end{array}$ & $\begin{array}{l}2.9 \\
2.0\end{array}$ & $\begin{array}{l}2.9 \\
2.0\end{array}$ \\
\hline Current balance & 4.8 & 5.8 & 5.3 & 3.3 & 1.9 & 3.2 & 4.6 & 6.1 & 6.6 & 6.7 \\
\hline Overall balance (excluding grants) & -6.4 & -1.1 & -1.9 & -6.0 & -10.7 & -8.5 & -3.9 & -2.5 & -2.3 & -2.2 \\
\hline Overall balance (including grants) & -6.1 & -1.0 & -1.1 & -4.0 & -6.3 & -6.1 & -3.0 & -1.7 & -1.4 & -1.4 \\
\hline Financing & 6.6 & 3.3 & 3.2 & 4.0 & 6.3 & 6.1 & 3.0 & 1.7 & 1.4 & 1.4 \\
\hline $\begin{array}{l}\text { External (net) } \\
\quad \text { Loans }\end{array}$ & $\begin{array}{l}2.3 \\
2.3\end{array}$ & $\begin{array}{l}-0.9 \\
-0.9\end{array}$ & $\begin{array}{l}0.1 \\
0.0\end{array}$ & $\begin{array}{l}1.4 \\
1.4\end{array}$ & $\begin{array}{l}0.9 \\
0.9\end{array}$ & $\begin{array}{l}2.6 \\
2.6\end{array}$ & $\begin{array}{l}1.5 \\
1.5\end{array}$ & $\begin{array}{l}0.8 \\
0.8\end{array}$ & $\begin{array}{l}0.7 \\
0.7\end{array}$ & $\begin{array}{l}0.7 \\
0.7\end{array}$ \\
\hline $\begin{array}{l}\text { Drawings } \\
\text { Of which: }\end{array}$ & 3.9 & 4.7 & 2.4 & 5.5 & 4.1 & 6.0 & 5.1 & 3.5 & 4.4 & 4.3 \\
\hline Identified hurricane financing & $\ldots$ & $\ldots$ & $\ldots$ & $\ldots$ & 1.5 & 2.3 & $\ldots$ & $\ldots$ & $\ldots$ & $\ldots$ \\
\hline Amortization & 1.6 & 5.5 & 2.4 & 4.1 & 3.3 & 3.3 & 3.6 & 2.7 & 3.7 & 3.6 \\
\hline Domestic financing & 4.2 & 4.1 & 3.2 & 2.6 & 5.4 & 3.4 & 1.5 & 0.8 & 0.7 & 0.7 \\
\hline ECCB (net) & 0.0 & -0.2 & -0.1 & 0.0 & 0.0 & 0.0 & 0.0 & 0.0 & 0.0 & 0.0 \\
\hline Commercial banks (net) & 2.6 & 2.7 & 0.1 & -2.6 & 0.6 & 1.4 & 0.4 & 0.2 & 0.2 & 0.2 \\
\hline Other domestic financing & 1.6 & 1.6 & 3.1 & 5.2 & 4.9 & 2.0 & 1.1 & 0.6 & 0.5 & 0.5 \\
\hline Statistical discrepancy & -0.4 & -2.3 & 3.9 & 0.0 & 0.0 & 0.0 & 0.0 & 0.0 & 0.0 & 0.0 \\
\hline Memorandum items: & & & & & & & & & & \\
\hline GDP (at market prices, in EC\$ millions) & 2,538 & 2,616 & 2,665 & 2,607 & 2,699 & 2,854 & 3,013 & 3,170 & 3,335 & 3,509 \\
\hline Debt service (in percent of current revenue) & 18.7 & 32.2 & 19.7 & 26.0 & 24.5 & 25.6 & 26.3 & 22.5 & 25.5 & 25.0 \\
\hline Central government debt (in percent of GDP) 2/ & 63.5 & 64.5 & 64.8 & 72.2 & 76.1 & 78.0 & 76.8 & 74.7 & 72.4 & 70.2 \\
\hline
\end{tabular}

Sources: St. Lucia authorities; and Fund staff estimates and projections.

1/ Data are for fiscal years beginning April 1.

$2 /$ Includes liabilities related to the construction and financing of public projects by the private sector. 
Table 4. St. Lucia: Balance of Payments Summary, 2006-15

\begin{tabular}{|c|c|c|c|c|c|c|c|c|c|c|}
\hline & \multirow[b]{2}{*}{2006} & \multirow[b]{2}{*}{2007} & \multirow[b]{2}{*}{2008} & \multirow[b]{2}{*}{2009} & \multirow{2}{*}{$\begin{array}{r}\text { Est. } \\
2010\end{array}$} & \multicolumn{5}{|c|}{ Projections } \\
\hline & & & & & & 2011 & 2012 & 2013 & 2014 & 2015 \\
\hline & \multicolumn{10}{|c|}{ (In millions of Eastern Caribbean dollars) } \\
\hline Current account & -761.5 & -810.7 & -747.3 & -373.3 & -443.5 & -714.3 & -542.8 & -480.5 & -530.9 & -559.4 \\
\hline Trade balance & $-1,089.8$ & $-1,161.6$ & -996.6 & -702.5 & -892.4 & $-1,220.9$ & $-1,081.0$ & $-1,068.9$ & $-1,225.8$ & $-1,306.4$ \\
\hline Exports f.o.b. & 316.9 & 301.0 & 447.4 & 516.5 & 471.6 & 412.9 & 435.8 & 461.7 & 488.9 & 512.2 \\
\hline Of which & & 1.7 & 2.2 & 2.3 & 1.5 & & & & & \\
\hline Bananas & 48.1 & 43.7 & 58.9 & 58.4 & 41.1 & 32.7 & 34.4 & 37.4 & 40.3 & 37.8 \\
\hline Manufactured exports & 64.6 & 71.6 & 79.1 & 76.1 & 74.6 & 76.2 & 78.8 & 81.5 & 84.4 & 87.2 \\
\hline Imports f.o.b. & $-1,406.6$ & $-1,462.6$ & $-1,444.0$ & $-1,219.0$ & $-1,364.0$ & $-1,633.8$ & $-1,516.8$ & $-1,530.6$ & $-1,714.7$ & $-1,818.6$ \\
\hline Services (net) & 446.4 & 497.7 & 399.9 & 439.1 & 571.6 & 634.6 & 674.2 & 730.7 & 842.8 & 900.7 \\
\hline Credits & 902.4 & $1,004.7$ & 981.7 & 952.1 & $1,088.6$ & $1,171.0$ & $1,230.6$ & $1,307.6$ & $1,441.1$ & $1,530.2$ \\
\hline Travel & 768.4 & 857.7 & 839.7 & 799.7 & 929.0 & 999.0 & $1,048.8$ & $1,116.3$ & $1,239.8$ & $1,318.4$ \\
\hline Other nonfactor services & 133.9 & 147.0 & 142.0 & 152.3 & 159.6 & 172.0 & 181.9 & 191.3 & 201.2 & 211.8 \\
\hline Debits & 456.0 & 506.9 & 581.7 & 513.0 & 517.0 & 536.4 & 556.4 & 576.9 & 598.2 & 629.5 \\
\hline Travel & 106.2 & 114.1 & 122.3 & 126.2 & 127.2 & 131.9 & 136.9 & 144.0 & 151.4 & 159.4 \\
\hline Other nonfactor services & 349.8 & 392.8 & 459.5 & 386.8 & 389.8 & 404.5 & 419.6 & 432.9 & 446.8 & 470.1 \\
\hline Income payments (net) & -150.4 & -183.5 & -194.5 & -143.4 & -157.3 & -164.4 & -174.6 & -182.9 & -190.6 & -198.5 \\
\hline Current transfers & 32.3 & 36.6 & 43.8 & 33.5 & 34.5 & 36.5 & 38.6 & 40.6 & 42.7 & 44.9 \\
\hline Net private transfers & 33.6 & 29.1 & 33.3 & 32.1 & 33.0 & 34.9 & 36.9 & 38.8 & 40.9 & 43.0 \\
\hline Net official transfers & -1.2 & 7.5 & 10.5 & 1.4 & 1.5 & 1.5 & 1.6 & 1.7 & 1.8 & 1.9 \\
\hline Capital and financial account & 868.8 & 845.8 & 633.9 & 459.7 & 436.0 & 609.1 & 572.2 & 495.5 & 553.6 & 579.7 \\
\hline Capital & 30.7 & 23.4 & 29.4 & 69.8 & 70.4 & 117.8 & 26.5 & 27.9 & 29.3 & 30.9 \\
\hline Financial (net) & 838.1 & 822.4 & 831.4 & 390.0 & 365.6 & 491.2 & 545.7 & 467.6 & 524.3 & 548.8 \\
\hline Official capital & 58.5 & -22.6 & -25.5 & 35.7 & -23.3 & 61.2 & 44.9 & 26.3 & 23.7 & 23.7 \\
\hline Commercial banks & 174.0 & 216.6 & 466.2 & -11.9 & 35.2 & 54.5 & 74.3 & -1.0 & 27.3 & 27.2 \\
\hline Private capital & 605.6 & 609.6 & 411.5 & 395.9 & 348.3 & 375.5 & 426.7 & 442.4 & 473.2 & 497.9 \\
\hline \multicolumn{11}{|l|}{ Of which: } \\
\hline Net direct investment & 631.6 & 734.1 & 411.5 & 395.9 & 348.3 & 375.5 & 426.8 & 442.5 & 473.1 & 497.9 \\
\hline Errors and omissions & -71.1 & 14.9 & 83.1 & 166.4 & 0.0 & 0.0 & 0.0 & 0.0 & 0.0 & 0.0 \\
\hline Overall balance & 36.3 & 50.0 & -30.4 & 79.9 & -7.5 & -105.3 & 29.4 & 14.9 & 22.7 & 20.3 \\
\hline Financing & -36.3 & -50.0 & 30.4 & -79.9 & 7.5 & 105.3 & -29.4 & -14.9 & -22.7 & -20.3 \\
\hline Change in imputed reserves (increase -) & -48.6 & -51.3 & 29.5 & -18.3 & 7.5 & -2.4 & -29.4 & -14.9 & -22.7 & -20.3 \\
\hline Change in govt. foreign assets & 12.3 & 1.3 & 0.9 & 0.0 & 0.0 & 0.0 & 0.0 & 0.0 & 0.0 & 0.0 \\
\hline Change in SDR Holdings & & & & -22.8 & & & & & & \\
\hline $\begin{array}{l}\text { Identified hurricane-related financing } \\
\text { Of which: }\end{array}$ & 0.0 & 0.0 & 0.0 & 0.0 & 0.0 & 107.7 & 0.0 & 0.0 & 0.0 & 0.0 \\
\hline \multirow[t]{2}{*}{ RCF/ENDA } & & & & & & 22.1 & & & & \\
\hline & \multicolumn{10}{|c|}{ (In percent of GDP) } \\
\hline \multicolumn{11}{|l|}{ Memorandum items: } \\
\hline Current account & -30.2 & -31.3 & -27.8 & -14.4 & -16.7 & -25.4 & -18.2 & -15.4 & -16.1 & -16.1 \\
\hline Exports f.o.b. & 12.6 & 11.6 & 16.6 & 20.0 & 17.7 & 14.7 & 14.7 & 14.8 & 14.9 & 14.8 \\
\hline Imports f.o.b. & -55.8 & -56.4 & -53.7 & -47.1 & -51.3 & -58.1 & -51.0 & -48.9 & -52.1 & -52.5 \\
\hline Net private transfers & 1.3 & 1.1 & 1.2 & 1.2 & 1.2 & 1.2 & 1.2 & 1.2 & 1.2 & 1.2 \\
\hline Foreign direct investment & 25.1 & 28.3 & 15.3 & 15.3 & 13.1 & 13.3 & 14.3 & 14.1 & 14.4 & 14.4 \\
\hline \multicolumn{11}{|l|}{$\begin{array}{l}\text { Indicators of diversification } \\
\text { (In percent of exports of goods and } \\
\text { nonfactor services) }\end{array}$} \\
\hline Banana exports & 3.9 & 3.3 & 4.1 & 4.0 & 2.6 & 2.1 & 2.1 & 2.1 & 2.1 & 1.9 \\
\hline Tourism receipts & 63.0 & 65.7 & 58.8 & 54.5 & 59.5 & 63.1 & 62.9 & 63.1 & 64.2 & 64.6 \\
\hline Tourism receipts & 30.5 & 33.1 & 31.2 & 30.9 & 34.9 & 35.5 & 35.3 & 35.7 & 37.7 & 38.1 \\
\hline Total trade & 68.4 & 68.1 & 70.3 & 67.0 & 69.0 & 72.7 & 65.6 & 63.7 & 66.9 & 67.3 \\
\hline Exports of goods and nonfactor services & 48.4 & 50.4 & 53.1 & 56.7 & 58.6 & 56.3 & 56.0 & 56.5 & 58.6 & 59.0 \\
\hline Imports of goods and nonfactor services & 73.9 & 76.0 & 75.3 & 66.9 & 70.7 & 77.1 & 69.7 & 67.3 & 70.3 & 70.7 \\
\hline Terms of trade for GNFS (percentage change) & -12.7 & -11.2 & -5.5 & 14.9 & -6.5 & -0.2 & 0.1 & 0.3 & 4.1 & 0.1 \\
\hline Excluding tourism (percentage change) & -6.3 & -7.2 & -0.6 & 25.6 & -6.2 & -4.9 & -3.8 & -1.8 & -2.0 & -1.8 \\
\hline Public sector external debt (end of period) & 44.8 & 41.8 & 36.5 & 39.7 & 39.5 & 40.0 & 39.4 & 38.3 & 37.1 & 35.9 \\
\hline
\end{tabular}

Sources: St. Lucia authorities; ECCB; and Fund staff estimates and projections. 
Table 5. St. Lucia: Monetary Survey, 2004-10

\begin{tabular}{|c|c|c|c|c|c|c|c|}
\hline & 2004 & 2005 & 2006 & 2007 & 2008 & 2009 & $\begin{array}{r}\text { Est. } \\
2010\end{array}$ \\
\hline & \multicolumn{7}{|c|}{ (In millions of Eastern Caribbean dollars) } \\
\hline Net foreign assets & 318.7 & 99.9 & 117.6 & -47.7 & -543.3 & -513.2 & -555.9 \\
\hline ECCB (imputed reserves) 1/ & 351.5 & 308.3 & 356.9 & 408.2 & 378.8 & 397.0 & 389.5 \\
\hline Commercial banks & -32.9 & -208.5 & -239.3 & -455.9 & -922.1 & -910.2 & -945.4 \\
\hline Net domestic assets & $1,190.5$ & $1,611.9$ & $1,938.6$ & $2,230.9$ & $3,009.9$ & $3,017.0$ & $3,129.3$ \\
\hline Public sector credit (net) & -324.7 & -275.9 & -222.8 & -650.6 & -549.5 & -548.3 & -525.4 \\
\hline Central government & -130.7 & -90.7 & -32.0 & 17.2 & 69.0 & 45.3 & 60.3 \\
\hline ECCB & -13.7 & -10.0 & -19.8 & -43.2 & 5.9 & -5.6 & -5.6 \\
\hline Commercial banks & -117.0 & -80.6 & -12.2 & 60.4 & 63.1 & 50.9 & 65.9 \\
\hline Net credit to rest of public sector & -194.0 & -185.2 & -190.8 & -667.9 & -618.6 & -593.6 & -585.6 \\
\hline National Insurance Corporation & -240.9 & -248.2 & -264.2 & -291.7 & -340.3 & -408.0 & -408.0 \\
\hline Other & 46.9 & 62.9 & 73.4 & -376.2 & -278.3 & -185.6 & -177.7 \\
\hline Credit to private sector & $1,650.6$ & $1,917.3$ & $2,386.8$ & $3,090.8$ & $3,680.6$ & $3,745.4$ & $3,793.3$ \\
\hline Net credit to nonbank financial inst. & -35.4 & -37.8 & -40.4 & -41.3 & -29.2 & -55.4 & -56.9 \\
\hline Other items (net) & -100.0 & 8.3 & -185.1 & -168.0 & -91.9 & -124.8 & -81.8 \\
\hline Broad money & $1,509.2$ & $1,711.8$ & $2,056.2$ & $2,183.2$ & $2,466.6$ & $2,503.8$ & $2,573.4$ \\
\hline Money & 481.3 & 547.3 & 560.7 & 639.0 & 661.4 & 652.6 & 670.7 \\
\hline Currency in circulation & 99.2 & 106.4 & 126.6 & 128.0 & 142.6 & 148.4 & 152.5 \\
\hline Demand deposits & 382.2 & 440.9 & 434.1 & 510.9 & 518.8 & 504.2 & 518.2 \\
\hline Quasi-money & $1,027.8$ & $1,164.5$ & $1,495.5$ & $1,544.2$ & $1,805.2$ & $1,851.2$ & $1,902.7$ \\
\hline Time deposits & 178.7 & 185.9 & 222.7 & 283.3 & 420.1 & 405.4 & 428.2 \\
\hline Savings deposits & 810.4 & 916.3 & $1,064.9$ & $1,150.8$ & $1,226.6$ & $1,301.7$ & $1,337.9$ \\
\hline \multirow[t]{2}{*}{ Foreign currency deposits } & 38.7 & 62.2 & 207.9 & 110.2 & 158.5 & 144.1 & 136.6 \\
\hline & \multicolumn{7}{|c|}{ (Annual percentage change) } \\
\hline Net foreign assets & -4.9 & -68.7 & 17.8 & -140.5 & $-1,040.0$ & 5.6 & 8.3 \\
\hline Net domestic assets & 15.0 & 35.4 & 20.3 & 15.1 & 34.9 & 0.2 & 3.7 \\
\hline Credit to private sector & 10.2 & 16.2 & 24.5 & 29.5 & 19.1 & 1.8 & 1.3 \\
\hline Broad money & 10.1 & 13.4 & 20.1 & 6.2 & 13.0 & 1.5 & 2.8 \\
\hline Money & 40.3 & 13.7 & 2.4 & 14.0 & 3.5 & -1.3 & 2.8 \\
\hline \multirow[t]{2}{*}{ Quasi-money 2/ } & 0.1 & 13.3 & 28.4 & 3.3 & 16.9 & 2.6 & 2.8 \\
\hline & \multicolumn{7}{|c|}{ (Percent contribution compared to $\mathrm{M} 2$ at the beginning of the year) } \\
\hline Net foreign assets & -1.2 & -14.5 & 1.0 & -8.0 & -22.7 & 1.2 & -1.7 \\
\hline Net domestic assets & 11.3 & 27.9 & 19.1 & 14.2 & 35.7 & 0.3 & 4.5 \\
\hline Public sector credit (net) & -2.5 & 3.2 & 3.1 & -20.8 & 4.6 & 0.0 & 0.9 \\
\hline Of which: central government & -1.9 & 2.7 & 3.4 & 2.4 & 2.4 & -1.0 & 0.6 \\
\hline Credit to private sector & 11.1 & 17.7 & 27.4 & 34.2 & 27.0 & 2.6 & 1.9 \\
\hline Net credit to nonbank financial inst. & 0.3 & -0.1 & -0.1 & 0.0 & 0.5 & -1.0 & -0.1 \\
\hline Other items (net) & 2.4 & 7.2 & -11.3 & 0.8 & 3.5 & -1.3 & 1.7 \\
\hline \multicolumn{8}{|l|}{ Memorandum items: } \\
\hline Income velocity of M2 3/ & 1.5 & 1.5 & 1.3 & 1.2 & 1.2 & 1.0 & 1.0 \\
\hline
\end{tabular}

Sources: St. Lucia authorities; ECCB; and Fund staff estimates and projections.

1/ Not including the IMF's SDR allocation of September 2009, in the amount of SDR 13.8 million (EC\$58.7 million).

2/ Including resident foreign currency deposits.

3/ Nominal GDP at market prices divided by liabilities to the private sector. 
Table 6. St. Lucia: Indicators of External and Financial Vulnerability, 2005-10 (Annual percentage changes, unless otherwise specified)

\begin{tabular}{|c|c|c|c|c|c|c|}
\hline & 2005 & 2006 & 2007 & 2008 & 2009 & $\begin{array}{r}\text { Est. } \\
2010\end{array}$ \\
\hline \multicolumn{7}{|l|}{ External indicators } \\
\hline Merchandise exports & -7.7 & 32.2 & -5.0 & 48.6 & 15.4 & -8.7 \\
\hline Merchandise imports & 20.1 & 24.6 & 4.0 & -1.3 & -15.6 & 11.9 \\
\hline Terms of trade deterioration (-) & -1.6 & -12.7 & -11.2 & -5.5 & 14.9 & -6.5 \\
\hline Tourism earnings & 9.3 & -20.0 & 11.6 & -2.1 & -4.8 & 16.2 \\
\hline Banana export earnings & -23.0 & 15.9 & -9.2 & 35.0 & -0.8 & -29.7 \\
\hline Current account balance (in percent of GDP) & -17.1 & -30.2 & -31.3 & -27.8 & -14.4 & -16.7 \\
\hline $\begin{array}{l}\text { Capital and financial account balance (in percent of GDP) 1/ } \\
\text { Of which }\end{array}$ & 15.9 & 34.5 & 32.6 & 23.6 & 17.8 & 16.4 \\
\hline Foreign direct investment (in percent of GDP) & 8.9 & 25.1 & 28.3 & 15.3 & 15.3 & 13.1 \\
\hline \multicolumn{7}{|l|}{ Gross international reserves of the ECCB } \\
\hline In millions of U.S. dollars & 600.8 & 696.0 & 764.5 & 759.0 & 800.8 & 818.2 \\
\hline In percent of broad money & 17.9 & 18.6 & 18.6 & 17.0 & 17.5 & 17.8 \\
\hline \multicolumn{7}{|l|}{ Gross imputed reserves } \\
\hline In millions of U.S. dollars & 114.2 & 132.2 & 151.2 & 140.3 & 147.0 & 144.3 \\
\hline In percent of short-term liablilities & $\ldots$ & $\ldots$ & $\ldots$ & $\ldots$ & $\ldots$ & $\ldots$ \\
\hline External public debt (in percent of GDP) & 47.4 & 44.8 & 41.8 & 36.5 & 39.7 & 39.5 \\
\hline \multicolumn{7}{|l|}{ External debt service (in percent of exports of goods and } \\
\hline nonfactor services) & 6.5 & 11.2 & 17.0 & 11.4 & 11.2 & 9.1 \\
\hline \multicolumn{7}{|l|}{ Of which } \\
\hline Interest & 2.2 & 7.3 & 9.9 & 3.4 & 5.2 & 3.3 \\
\hline $\begin{array}{l}\text { Nominal exchange rate (EC dollars per U.S. dollar, } \\
\text { end period) }\end{array}$ & 2.7 & 2.7 & 2.7 & 2.7 & 2.7 & 2.7 \\
\hline Real effective exchange rate depreciation (-), end period & -0.4 & 0.3 & -3.8 & 0.5 & 3.2 & $\cdots$ \\
\hline \multicolumn{7}{|l|}{ Financial indicators } \\
\hline Broad money & 13.4 & 20.1 & 6.2 & 13.0 & 1.5 & 2.8 \\
\hline Credit to the private sector & 16.2 & 24.5 & 29.5 & 19.1 & 1.8 & 1.3 \\
\hline \multicolumn{7}{|l|}{ Prudential indicators (in percent) } \\
\hline Capital adequacy ratio (local banks) & 14.4 & 17.6 & 20.2 & 15.6 & 20.8 & $\ldots$ \\
\hline NPLs to total loans ratio & 12.6 & 8.5 & 5.8 & 6.6 & 8.3 & $\ldots$ \\
\hline \multicolumn{7}{|l|}{ Of which } \\
\hline Local banks & 16.5 & 10.5 & 7.3 & 9.0 & 10.2 & $\ldots$ \\
\hline Foreign banks & 9.9 & 7.1 & 4.8 & 5.0 & 7.4 & $\ldots$ \\
\hline Loan loss provision to NPLs ratio & 40.4 & 40.1 & 45.5 & 37.5 & 29.3 & $\ldots$ \\
\hline \multicolumn{7}{|l|}{ Of which } \\
\hline Local banks & 44.8 & 35.2 & 38.6 & 27.8 & 20.1 & $\ldots$ \\
\hline Foreign banks & 35.4 & 45.2 & 52.2 & 48.6 & 35.9 & $\ldots$ \\
\hline Gross government claims to total assets ratio & 14.6 & 11.8 & 10.0 & 9.7 & 9.6 & $\ldots$ \\
\hline Foreign currency deposits to total deposits ratio & 3.0 & 9.0 & 5.6 & 6.3 & 6.4 & $\ldots$ \\
\hline Net foreign currency exposure to capital (local banks) & 122.3 & 53.6 & 73.9 & 49.3 & 47.4 & $\ldots$ \\
\hline Contingent liabilities to capital (local banks) & 149.5 & 124.3 & 78.3 & 104.3 & 60.0 & $\ldots$ \\
\hline (Pre-tax) return on average assets & 2.3 & 2.4 & 2.8 & 3.2 & 0.5 & $\ldots$ \\
\hline Yield to maturity sovereign bonds 2 / & 6.5 & 7.1 & 7.5 & 7.5 & 7.2 & $\ldots$ \\
\hline
\end{tabular}

Sources: St. Lucia authorities; ECCB; and Fund staff estimates and projections.

$1 /$ Includes errors and omissions.

2/ Composite index, including RGSM bonds. 
Table 7. St. Lucia: Indicators of Capacity to Repay the Fund, 2010-21 1/

\begin{tabular}{|c|c|c|c|c|c|c|c|c|c|c|c|c|}
\hline & \multirow{2}{*}{$\begin{array}{r}\text { Est. } \\
2010\end{array}$} & \multicolumn{11}{|c|}{ Projections } \\
\hline & & 2011 & 2012 & 2013 & 2014 & 2015 & 2016 & 2017 & 2018 & 2019 & 2020 & 2021 \\
\hline Fund obligations based on existing credit (in millions of SDRs) & 0.00 & 0.00 & 0.02 & 0.02 & 0.02 & 1.40 & 1.39 & 1.39 & 1.38 & 1.38 & 0.00 & 0.00 \\
\hline Principal & 0.00 & 0.00 & 0.00 & 0.00 & 0.00 & 1.38 & 1.38 & 1.38 & 1.38 & 1.38 & 0.00 & 0.00 \\
\hline Charges and interest & 0.00 & 0.00 & 0.02 & 0.02 & 0.02 & 0.02 & 0.01 & 0.01 & 0.00 & 0.00 & 0.00 & 0.00 \\
\hline \multicolumn{13}{|l|}{ Fund obligations based on existing and prospective credit } \\
\hline (in millions of SDRs) & 0.00 & 0.02 & 0.05 & 0.05 & 0.62 & 2.17 & 1.97 & 2.16 & 2.15 & 2.14 & 0.77 & 0.38 \\
\hline Principal & 0.00 & 0.00 & 0.00 & 0.00 & 0.57 & 2.14 & 1.95 & 2.14 & 2.14 & 2.14 & 0.77 & 0.38 \\
\hline Charges and interest & 0.00 & 0.02 & 0.05 & 0.05 & 0.05 & 0.03 & 0.02 & 0.02 & 0.01 & 0.00 & 0.00 & 0.00 \\
\hline \multirow{2}{*}{\multicolumn{13}{|c|}{$\begin{array}{l}\text { Fund credit outstanding based on existing and prospective credit (in } \\
\text { millions of SDRs) }\end{array}$}} \\
\hline & 6.89 & 12.25 & 12.25 & 12.25 & 11.68 & 9.53 & 7.58 & 5.44 & 3.29 & 1.15 & 0.38 & 0.00 \\
\hline \multicolumn{13}{|l|}{ Total obligations based on existing and prospective credit } \\
\hline In millions of U.S. dollars $2 /$ & 0.00 & 0.03 & 0.08 & 0.08 & 0.95 & 3.31 & 3.01 & 3.30 & 3.28 & 3.27 & 1.17 & 0.58 \\
\hline In percent of exports of goods and services & 0.00 & 0.01 & 0.01 & 0.01 & 0.13 & 0.44 & 0.38 & 0.39 & 0.37 & 0.35 & 0.12 & 0.06 \\
\hline In percent of external debt service $3 /$ & 0.00 & 0.05 & 0.12 & 0.14 & 1.36 & 4.66 & 3.95 & 4.08 & 3.84 & 3.53 & 1.19 & 0.57 \\
\hline In percent of GDP & 0.00 & 0.00 & 0.01 & 0.01 & 0.10 & 0.32 & 0.28 & 0.29 & 0.28 & 0.26 & 0.09 & 0.04 \\
\hline In percent of quota & 0.00 & 0.13 & 0.33 & 0.33 & 4.05 & 14.18 & 12.88 & 14.12 & 14.05 & 13.99 & 5.03 & 2.48 \\
\hline In percent of net international reserves & 0.00 & 0.02 & 0.05 & 0.05 & 0.56 & 1.87 & 1.62 & 1.68 & 1.59 & 1.51 & 0.52 & 0.24 \\
\hline \multicolumn{13}{|l|}{ Outstanding Fund credit $3 /$} \\
\hline In millions of U.S. dollars $2 /$ & 10.51 & 18.69 & 18.69 & 18.69 & 17.82 & 14.54 & 11.57 & 8.30 & 5.02 & 1.75 & 0.58 & 0.00 \\
\hline In percent of exports of goods and services & 1.82 & 3.19 & 3.03 & 2.85 & 2.49 & 1.92 & 1.46 & 0.99 & 0.57 & 0.19 & 0.06 & 0.00 \\
\hline In percent of external debt service $3 /$ & 19.93 & 33.41 & 29.79 & 34.18 & 25.63 & 20.45 & 15.19 & 10.28 & 5.87 & 1.90 & 0.59 & 0.00 \\
\hline In number of months of imports of goods and services & 0.18 & 0.28 & 0.29 & 0.29 & 0.25 & 0.19 & 0.14 & 0.10 & 0.06 & 0.02 & 0.01 & 0.00 \\
\hline In percent of GDP & 1.32 & 2.22 & 2.10 & 2.00 & 1.81 & 1.41 & 1.07 & 0.73 & 0.42 & 0.14 & 0.04 & 0.00 \\
\hline In percent of quota & 45.03 & 80.07 & 80.07 & 80.07 & 76.34 & 62.29 & 49.54 & 35.56 & 21.50 & 7.52 & 2.48 & 0.00 \\
\hline In percent of net international reserves & 7.29 & 12.88 & 11.98 & 11.57 & 10.48 & 8.19 & 6.21 & 4.24 & 2.44 & 0.81 & 0.25 & 0.00 \\
\hline Net use of Fund credit (in millions of SDRs) & 0.00 & 5.36 & 0.00 & 0.00 & -0.57 & -2.14 & -1.95 & -2.14 & -2.14 & -2.14 & -0.77 & -0.38 \\
\hline Disbursements & 0.00 & 5.36 & 0.00 & 0.00 & 0.00 & 0.00 & 0.00 & 0.00 & 0.00 & 0.00 & 0.00 & 0.00 \\
\hline Repayments and Repurchases & 0.00 & 0.00 & 0.00 & 0.00 & 0.57 & 2.14 & 1.95 & 2.14 & 2.14 & 2.14 & 0.77 & 0.38 \\
\hline \multicolumn{13}{|l|}{ Memorandum items: } \\
\hline Nominal GDP (in millions of U.S. dollars) & 797.0 & 843.1 & 890.1 & 934.9 & 982.1 & 1032.0 & 1084.5 & 1135.6 & 1189.5 & 1245.9 & 1305.0 & 1366.9 \\
\hline Exports of goods and services (in millions of U.S. dollars) & 577.9 & 586.6 & 617.2 & 655.3 & 714.8 & 756.4 & 794.4 & 835.7 & 879.5 & 926.3 & 976.0 & 1029.0 \\
\hline External debt service (in millions of U.S. dollars) $3 /$ & 52.7 & 55.9 & 62.7 & 54.7 & 69.5 & 71.1 & 76.1 & 80.7 & 85.5 & 92.4 & 98.6 & 101.2 \\
\hline Imports of goods and services (in millions of U.S. dollars) & 696.6 & 803.8 & 767.9 & 780.5 & 856.6 & 906.7 & 978.3 & 1017.9 & 1071.9 & 1127.7 & 1187.0 & 1248.5 \\
\hline Net imputed international reserves (in millions of U.S. dollars) & 144.3 & 145.2 & 156.0 & 161.6 & 170.0 & 177.5 & 186.1 & 195.6 & 205.9 & 216.6 & 227.9 & 239.8 \\
\hline
\end{tabular}

Sources: Fund staff estimates and projections.

1/ Assumes RCF/ENDA access in the amount of SDR 5.36 million (35 percent of quota).

$2 /$ US $\$ 1=0.655404$ SDR (as of November 30, 2010)

$3 /$ Including prospective repurchases/repayments. 


\section{Letter of Intent}

Castries, St. Lucia

December 21, 2010

Mr. Dominique Strauss-Kahn

Managing Director

International Monetary Fund

Washington, D.C. 20431

Dear Mr. Strauss-Kahn,

The St. Lucian economy has been adversely affected by Hurricane Tomas, which hit the island October 30, 2010. Torrential rains have resulted in landslides, causing human causalities and severe damage to the island's economic infrastructure. High winds and severe flooding have also damaged the agricultural sector (in particular the banana industry), as well as commercial and residential buildings. Fortunately, the impact on St. Lucia's important tourism sector was more limited. The initial estimate of the overall damage is about US\$336 million (33.6 percent of GDP), including loss of export earnings of some US\$45 million (5 percent of GDP). Our preliminary estimates suggest that real GDP growth will reach only about 0.5 percent of GDP in 2010, compared with a pre-hurricane estimate of 1.7 percent. Reflecting higher construction-related imports and a reduction in banana exports, the external current account deficit is projected to increase.

The government has moved quickly to provide immediate relief to those affected by the natural disaster, and initiated critical repair and reconstruction work financed by reallocating expenditure and using US\$3.2 million from the Caribbean Catastrophic Risk Insurance Fund. Many development partners have already indicated their support (including the Caribbean Development Bank, the World Bank, the European Union, and the CARICOM Development Fund), but the reconstruction process will require a considerable amount of time and resources. Accordingly, the government of St. Lucia requests emergency financing from the IMF amounting to SDR 5.36 million (US\$8.22 million), equivalent to 35 percent of quota, consisting of a disbursement of SDR 3.83 (US\$5.87 million) under the Rapid Credit Facility and a purchase equivalent to SDR 1.53 million (US\$2.35 million) under Emergency Natural Disaster Assistance. The IMF assistance will help meet the immediate foreign exchange needs stemming from the disaster, thereby maintaining confidence in the external position.

The Government is dealing with the effects of the crisis on several fronts. In terms of immediate priorities we have started to repair critical infrastructure (water distribution systems, roads, and bridges) and are providing assistance to those who suffered an abrupt loss of livelihoods. Going forward - and with the help of our donor countries and multilateral agencies - we will start with the reconstruction and rehabilitation and the development of a comprehensive economic and social program aimed at reactivating the economy and addressing the large social needs. The infrastructure needs are significant, but it is the 
government's intention to keep commercial borrowing to a minimum by limiting the financing of the rehabilitation and reconstruction effort to available grants and concessional financing. All cash disbursements received from donors will be channeled through the consolidated fund to ensure adequate accounting and use of these resources. While the fiscal deficit will temporarily increase to 6.3 percent of GDP in FY 2010/11 and 6.1 percent in FY 2011/12, we remain committed to achieving a debt-to-GDP target of 60 percent by 2020 , for which we will target a primary surplus of 2.9 percent of GDP after FY 2012/13. This target is 1.2 percentage point of GDP higher than the average under the active scenario discussed during the Article IV. To this end, we intend to implement both revenue and expenditure measures. On the revenue front, Cabinet has recently approved legislation to implement a market-based property tax, and we will seek its parliamentary approval with the view to making it effective by early FY 2011/12. Also, we are committed to introducing a VAT by April 2012 and have already initiated the consultative process with the private sector and other key stakeholders. On the expenditure side, and within the context of a broader civil service reform, we intend to freeze the civil service wage bill at 11 percent of GDP in the medium term and are taking steps to introduce a medium-term expenditure framework.

The government attaches great importance to implementing its agenda of structural reforms aimed at fostering private-sector-led economic growth and reducing poverty levels, consistent with our Millennium Development Goals. In this context, the government will continue to improve the investment climate, including by setting up a "one-stop-shop" for investors and by seeking passage of the Single Regulatory Unit and credit union legislations to strengthen supervision of the nonbanking sector and facilitate a more efficient allocation of capital.

It is hoped that the international financial community will support our efforts to restore economic growth and repair and rehabilitate our severely damaged social and economic infrastructure. We look forward to an early approval of financial assistance by the IMF and have identified financing gaps that could possibly be filled by our development partners and other multilateral agencies.

The government intends to continue to maintain a close policy dialogue with the Fund in an effort to strengthen St. Lucia's balance of payments situation and maintain macroeconomic stability. The government does not intend to impose new or intensify existing restrictions on the making of payments and transfers for current international transactions, introduce new or intensify existing trade restrictions for balance of payments purposes, or enter into bilateral payments agreements which are inconsistent with Article VIII of the Fund's Articles of Agreement.

Sincerely yours,

/s/

HON. STEPHENSON KING

PRIME MINISTER AND MINISTER

FOR FINANCE AND PLANNING 


\title{
INTERNATIONAL MONETARY FUND
}

\author{
ST. LUCIA \\ External and Public Debt Sustainability Analysis \\ Prepared by the Staff of the International Monetary Fund
}

December 23, 2010

This debt sustainability analysis (DSA) assesses the sustainability of St. Lucia's public and external debt. The analysis indicates that, under the baseline scenario discussed in this staff report, public debt will resume a sustainable trajectory in the medium term, barring further external shocks such as the natural disaster that hit the country recently. This sustainable debt trajectory, however, hinges on the successful fiscal consolidation to achieve fiscal primary surplus of 2.9 percent of GDP and the real GDP growth of 3.0 percent in the medium term. The risk of external debt distress remains moderate.

\section{INTRODUCTION}

1. St. Lucia has been significantly impacted by the 2008-09 global economic and financial crises, as the tourism demand from the main source economies declined on weak employment and consumption. Economic activities contracted by about 3.6 percent in 2009 after expanding on an average by about 3 percent in 2004-08. The primary balance turned to a deficit of 0.5 percent of GDP in 2009 from a surplus of 2.3 percent in 2008 , reflecting the counter-cyclical measures taken to cushion the impact of the crisis. ${ }^{1}$ Reflecting the weak growth and the fiscal deterioration, gross public debt increased from $661 / 2$ percent of GDP in 2008 to 73.9 percent in 2009. External debt constitutes a little over half of the public debt, however, the share of domestic debt is expanding, increasing by 5 percentage points to 34.4 percent of GDP in 2009. While the economy was on a path for a gradual recovery in 2010 led by tourism sector, St. Lucia was hit hard by Hurricane Tomas, resulting in a projected reduction in the real GDP growth by 1.2 percentage point from the pre-hurricane growth for 2010 to 0.5 percent post-hurricane.

\section{UNDERLYING DSA ASSUMPTIONS}

2. The DSA analysis is based on the following macroeconomic framework, assuming that the authorities will implement the near-term policies agreed with staff.

\footnotetext{
${ }^{1}$ The fiscal year starts April 1.
} 
- Growth and Inflation: Despite the impact of Hurricane Tomas and its damage to the agricultural production and infrastructure, the real GDP is projected to grow moderately by 0.5 percent in 2010 . A rebound of 4.1 percent growth is projected in 2011 , led by the reconstruction activities, and projected to average around 3.0 percent in the medium term. Inflation is expected to remain low at around 2 percent, anchored by the currency board arrangement.

\section{Box 1. Macroeconomic assumptions under the Baseline Scenario (2011-2030)}

- Following a prolonged slowdown in the aftermath of the global recession and the weak outlook of the employment and consumption in the major trading partners, real GDP growth is projected to average around 3.0 percent in the medium term. Inflation is expected to remain in low single digits, anchored by the currency board arrangement.

- The primary balance of the central government (including grants) is projected to improve to about 2.9 percent of GDP, reflecting the yield from the introduction of VAT in the first half of 2012. Also, civil service reform is assumed to contribute to reducing the wage bills by close to 2 percent of GDP to 11 percent of GDP in the medium term.

- The overall deficit is assumed to be financed roughly equally by domestic and external sources. Interest rates of 6.8 percent and 5.3 percent are assumed for domestic and external borrowings, respectively, in line with the historical average.

- Capital grants are conservatively projected at 0.9 percent of GDP per year, after the inflow above the historical levels in 2010/11 and 2011/12 for the support of the reconstruction from the damage of Hurricane Tomas. Capital expenditure is projected to converge to around 9.0 percent of GDP and stay constant over the medium term.

- FDI inflow is assumed to recover to around 14.4 percent of GDP, in line with historical average, following the sharp decline in 2008-2009 due to the global downturn. The current account deficit is projected to stay around 16 percent of GDP over the medium term.

- $\quad$ Fiscal Balance: The primary balance is projected to worsen temporarily to a deficit of around 2 percent of GDP in 2010 and 2011, as the impact of hurricane on revenue and the increase in capital expenditure for the reconstruction is only partially offset by higher grants. The primary surplus is assumed to improve over the medium term to an average of 2.9 percent of GDP, as planned policy measures would yield results, including the introduction of a market-based property tax in 2011, a VAT in the first half of 2012, and civil service reform to reduce wage bills to around 11 percent of GDP in the medium term. In the short term, the increase in the deficit will be limited to the identified sources of concessional financing. However, in the case the disbursements of the identified financing were to be delayed, the authorities might temporarily resort to borrowing in the Regional Government Securities Market (RGSM) or issue bonds outside of the region. In the medium term, the overall deficit is assumed to be financed mainly on market terms, and the interest rates of 
6.8 percent for domestic debt and 5.3 percent for external debt are assumed, in line with the historical average. As the new borrowings are assumed to be contracted largely on market terms reflecting the historical debt composition, the overall DSA results will not be altered should the authorities resort to bridge financing via the RGSM or a bond issue outside of the region.

- External Sector: The current account deficit is projected to widen in 2011 primarily due to the increase in import for the reconstruction, before converging to around 16 percent of GDP over the medium term. Tourism receipt is assumed to recover, in line with the strong growth in tourist arrivals before the hurricane. FDI inflows are projected to recover to historical levels of 14.4 percent of GDP, but remain below the recent peak of 2006-2007.

\section{Evaluation of Public Sector Debt Sustainability}

3. The debt-to-GDP ratio rose by $7 \frac{1}{2}$ percentage points to 73.9 percent in 2009 as a result of a recession and counter-cyclical fiscal policies. The ratio is projected to increase further by another 5.6 percentage points over the next two years to 79.5 percent in 2011 , reflecting the increase in capital expenditure for the reconstruction. In subsequent years, however, yields from the introduction of VAT and strengthened expenditure controls would contribute to the improvement in fiscal balances and put the public debt to a declining path over the medium term. The public debt is projected to fall to 59.9 percent of GDP by 2020 , achieving the Eastern Caribbean Central Bank (ECCB)'s benchmark of 60 percent by 2020.

\section{Sensitivity analysis shows that the public debt is most responsive to a shock to} real GDP growth. Under this scenario, which assumes the reduction of real GDP growth by one standard deviation below the historical average in 2011 and 2012, the PV of public debt increases to 121.2 percent of GDP in 2030 (Table 2a, Scenario B1). The combined shock of annual growth and the primary balance below historical averages would push the PV of public debt-to-GDP to 97.6 percent (Table 2a, Scenario B3). These results highlight St. Lucia's vulnerability to natural disasters and the risks of its high level of debt.

\section{Evaluation of External Debt Sustainability}

5. St. Lucia's external debt sustainability analysis includes only public sector debts due to the limitations in the data on private sector external borrowing. Under the baseline scenario, the PV of external debt is projected to increase to 40.0 percent of GDP in 2011 reflecting the widening fiscal deficit due to the impact of the hurricane. The ratio is 
projected to decline to 17.8 percent of GDP by 2030 , well below the prudential threshold of 50 percent $^{2}$ ( 1 and Table 3a).

6. Sensitivity analysis shows that the level of external debt is most responsive to an extreme shock of nominal exchange rate depreciation. The stress test assuming a one-time 30 percent nominal depreciation relative to the baseline in 2011 indicates that the PV of external debt-to-GDP ratio would rise to 56.8 percent and breach the threshold of 50 percent (Table 3b, Scenario B6). The debt service-to-export ratio rises to 17.1 percent under the most extreme export shock scenario assuming the export growth at one standard deviation below the historical average in 2010-11, below the prudential threshold of 25 percent.

\section{Conclusion}

7. Staff analysis shows that, under the baseline scenario, imbalances for the overall public sector would be on a declining and sustainable path, achieving the ECCB's debtto-GDP ratio target of 60 percent by 2020 . St. Lucia would then continue to reduce its stock of public debt steadily to 35.5 percent by 2030 . The main risks to the debt trajectory are the delay in implementation of measures to improve fiscal balances and shocks to economic growth including natural disaster.

8. External debt risk remains moderate. While the baseline scenario indicates no breach of any threshold over the projection period, the most extreme shock scenarios suggest breach of the PV of debt-to-GDP threshold and moderate increase of the PV of debt serviceto-export. It should be noted that the external debt sustainability analysis is constrained by the data limitation on private sector external borrowing.

9. The sustainable debt trajectory presented in the analysis is based on a strong fiscal adjustment and real GDP growth over the medium term. The government is assumed to successfully implement policy measures to achieve fiscal primary surplus of 2.9 percent of GDP, and the real GDP to grow by 3.0 percent in the medium term. As indicated by the stress tests, the public debt could take an unsustainable path should there be shortcomings in the fiscal consolidation and/or economic growth underperform.

\footnotetext{
${ }^{2}$ The DSA uses policy-dependent external debt burden indicators. Policy performance is measured by the Country Policy and Institutional Assessment Index (CPIA) compiled annually by the World Bank, categorizing countries into three groups based on the quality of their macroeconomic policies (strong, medium, and poor). St. Lucia is classified as a strong performer, with the thresholds on PV of debt-to-GDP, debt-to-exports and debt-to-revenue of 50, 200 and 300 percent respectively.
} 
Figure 1. St. Lucia: Indicators of Public and Publicly Guaranteed External Debt under Alternatives Scenarios, 2010-2030 1/
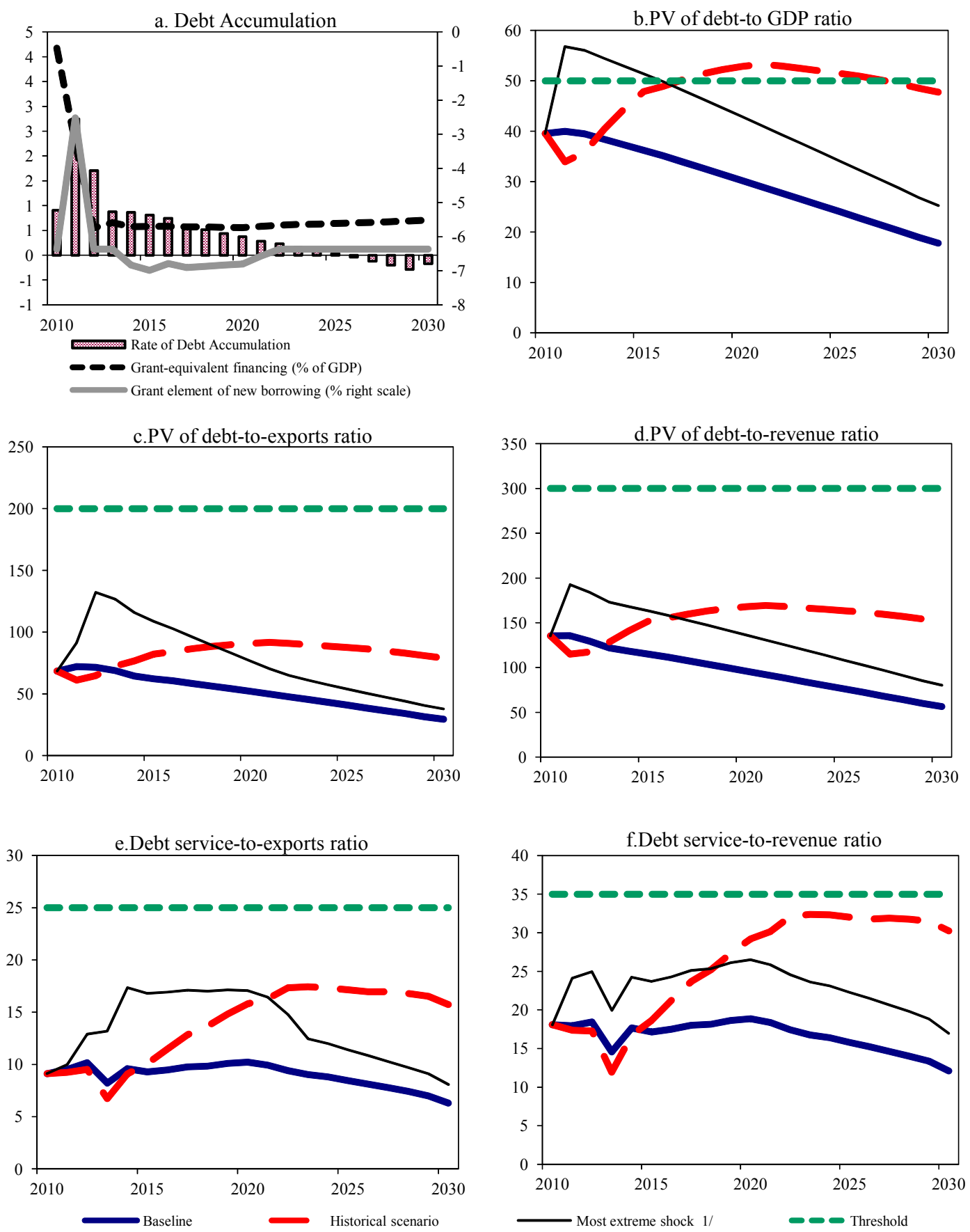

Sources: Country authorities; and staff estimates and projections.

1/ The most extreme stress test is the test that yields the highest ratio in 2020. In figure b. it corresponds to a One-time depreciation shock; in c. to a Exports shock; in d. to a One-time depreciation shock; in e. to a Exports shock and in figure f. to a One-time depreciation shock 
Figure 2.St. Lucia: Indicators of Public Debt Under Alternative Scenarios, 2010-2030 1/
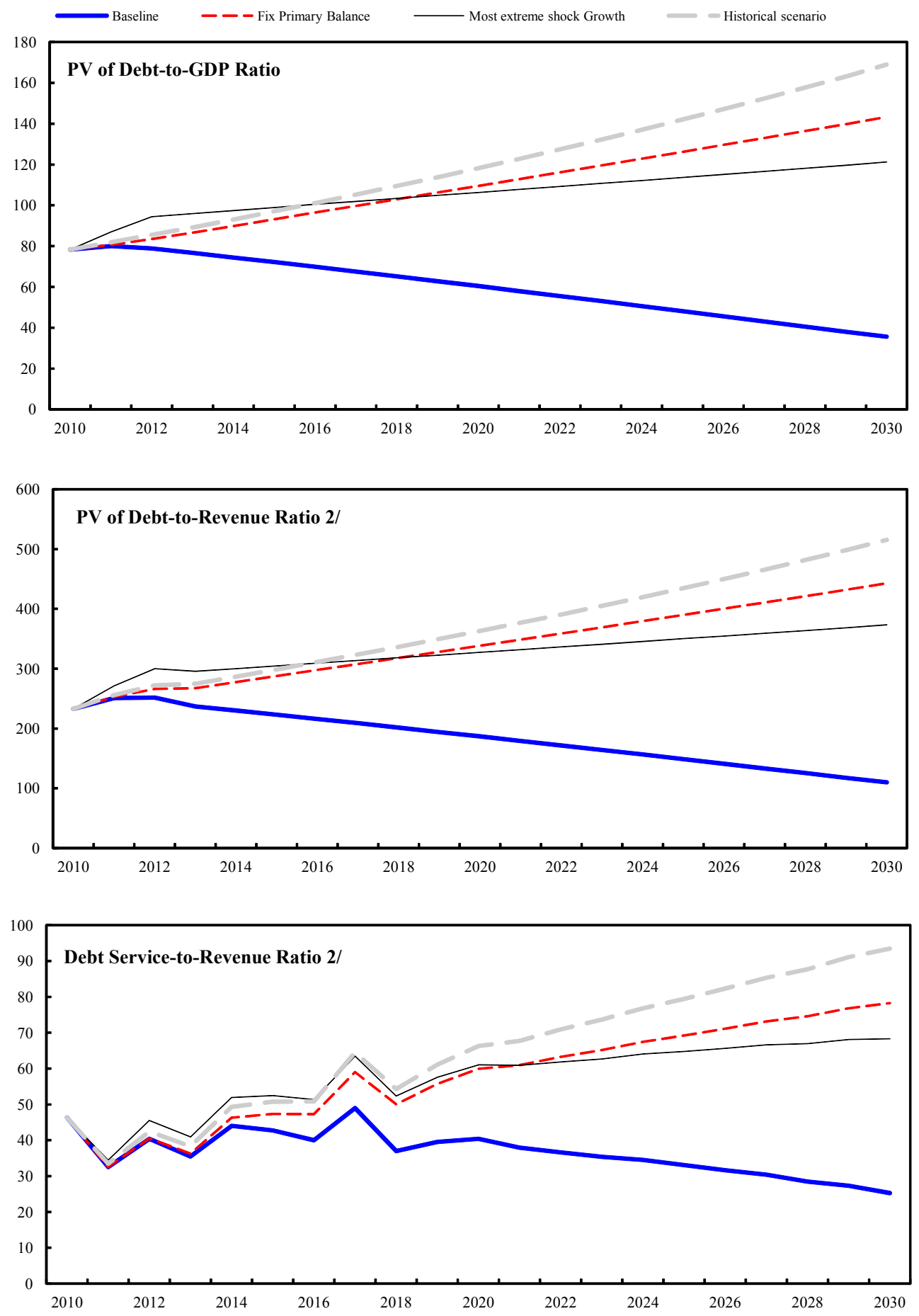

Sources: Country authorities; and staff estimates and projections.

1/ The most extreme stress test is the test that yields the highest ratio in 2020.

$2 /$ Revenues are defined inclusive of grants. 
Table 1a.St. Lucia: Public Sector Debt Sustainability Framework, Baseline Scenario, 2007-2030

(In percent of GDP, unless otherwise indicated)

\begin{tabular}{|c|c|c|c|c|c|c|c|c|c|c|c|c|c|c|c|}
\hline & \multicolumn{3}{|c|}{ Actual } & \multirow[b]{2}{*}{ Average } & \multirow[b]{2}{*}{$\begin{array}{l}\text { Standard } \\
\text { Deviation } \\
\end{array}$} & \multicolumn{5}{|l|}{ Estimate } & \multicolumn{5}{|c|}{ Projections } \\
\hline & 2007 & 2008 & 2009 & & & 2010 & 2011 & 2012 & 2013 & 2014 & 2015 & $\begin{array}{l}2010-15 \\
\text { Average } \\
\end{array}$ & 2020 & 2030 & $\begin{array}{c}2016-30 \\
\text { Average }\end{array}$ \\
\hline $\begin{array}{l}\text { Public sector debt } 1 / \\
\text { o/w foreign-currency denominated }\end{array}$ & $\begin{array}{l}66.5 \\
41.4\end{array}$ & $\begin{array}{l}66.4 \\
36.9\end{array}$ & $\begin{array}{l}73.9 \\
39.4\end{array}$ & & & $\begin{array}{l}77.7 \\
39.0\end{array}$ & $\begin{array}{l}79.5 \\
39.5\end{array}$ & $\begin{array}{l}78.3 \\
38.9\end{array}$ & $\begin{array}{l}76.1 \\
37.9\end{array}$ & $\begin{array}{l}73.9 \\
36.8\end{array}$ & $\begin{array}{l}71.6 \\
35.7\end{array}$ & & $\begin{array}{l}59.9 \\
29.8\end{array}$ & $\begin{array}{l}35.5 \\
17.7\end{array}$ & \\
\hline Change in public sector debt & 0.8 & 0.0 & 7.5 & & & 3.8 & 1.8 & -1.2 & -2.1 & -2.3 & -2.2 & & -2.4 & -2.4 & \\
\hline Identified debt-creating flows & -1.0 & -0.1 & 5.5 & & & 3.8 & 1.8 & -1.2 & -2.2 & -2.3 & -2.3 & & -2.3 & -2.6 & \\
\hline Primary deficit & -2.6 & -2.3 & 0.6 & 1.4 & 2.5 & 2.4 & 1.9 & -1.4 & -2.7 & -2.9 & -2.9 & -0.9 & -2.9 & -2.9 & -2.9 \\
\hline Revenue and grants & 28.4 & 30.6 & 31.0 & & & 33.6 & 31.9 & 31.4 & 32.4 & 32.4 & 32.4 & & 32.4 & 32.4 & \\
\hline of which: grants & 0.2 & 0.8 & 2.0 & & & 4.4 & 2.4 & 0.9 & 0.9 & 0.9 & 0.9 & & 0.9 & 0.9 & \\
\hline Primary (noninterest) expenditure & 25.8 & 28.3 & 31.6 & & & 36.0 & 33.7 & 30.0 & 29.6 & 29.5 & 29.5 & & 29.5 & 29.5 & \\
\hline Automatic debt dynamics & 1.6 & 2.2 & 4.9 & & & 1.4 & 0.0 & 0.2 & 0.5 & 0.5 & 0.6 & & 0.6 & 0.3 & \\
\hline Contribution from interest rate/growth differential & 1.0 & 1.8 & 5.1 & & & 2.2 & 0.1 & 0.4 & 0.8 & 0.7 & 0.7 & & 0.7 & 0.4 & \\
\hline of which: contribution from average real interest rate & 1.9 & 2.2 & 2.6 & & & 2.6 & 3.2 & 3.1 & 3.1 & 2.9 & 2.9 & & 2.5 & 1.5 & \\
\hline of which: contribution from real GDP growth & -1.0 & -0.5 & 2.5 & & & -0.4 & -3.1 & -2.7 & -2.3 & -2.2 & -2.2 & & -1.8 & -1.1 & \\
\hline Contribution from real exchange rate depreciation & 0.6 & 0.4 & -0.2 & & & -0.8 & -0.1 & -0.3 & -0.2 & -0.2 & -0.1 & & & $\ldots$ & \\
\hline Other identified debt-creating flows & 0.0 & 0.0 & 0.0 & & & 0.0 & 0.0 & 0.0 & 0.0 & 0.0 & 0.0 & & 0.0 & 0.0 & \\
\hline Privatization receipts (negative) & 0.0 & 0.0 & 0.0 & & & 0.0 & 0.0 & 0.0 & 0.0 & 0.0 & 0.0 & & 0.0 & 0.0 & \\
\hline Recognition of implicit or contingent liabilities & 0.0 & 0.0 & 0.0 & & & 0.0 & 0.0 & 0.0 & 0.0 & 0.0 & 0.0 & & 0.0 & 0.0 & \\
\hline Debt relief (HIPC and other) & 0.0 & 0.0 & 0.0 & & & 0.0 & 0.0 & 0.0 & 0.0 & 0.0 & 0.0 & & 0.0 & 0.0 & \\
\hline Other (specify, e.g. bank recapitalization) & 0.0 & 0.0 & 0.0 & & & 0.0 & 0.0 & 0.0 & 0.0 & 0.0 & 0.0 & & 0.0 & 0.0 & \\
\hline Residual, including asset changes & 1.8 & 0.1 & 2.0 & & & 0.0 & 0.0 & 0.0 & 0.1 & 0.1 & 0.1 & & -0.1 & 0.2 & \\
\hline PV of public sector debt & $\ldots$ & $\ldots$ & 74.5 & & & 78.3 & 80.0 & 78.9 & 76.7 & 74.4 & 72.2 & & 60.4 & 35.6 & \\
\hline $\mathrm{o} / \mathrm{w}$ foreign-currency denominated & $\ldots$ & $\ldots$ & 40.1 & & & 39.6 & 40.0 & 39.5 & 38.4 & 37.3 & 36.2 & & 30.2 & 17.8 & \\
\hline $\mathrm{o} / \mathrm{w}$ external & $\ldots$ & $\ldots$ & 40.1 & & & 39.6 & 40.0 & 39.5 & 38.4 & 37.3 & 36.2 & & 30.2 & 17.8 & \\
\hline $\mathrm{PV}$ of contingent liabilities (not included in public sector debt) & $\ldots$ & $\ldots$ & $\ldots$ & & & $\ldots$ & $\ldots$ & $\ldots$ & $\ldots$ & $\ldots$ & $\ldots$ & & $\ldots$ & ... & \\
\hline Gross financing need $2 /$ & 7.4 & 6.6 & 16.6 & & & 25.8 & 20.2 & 14.0 & 13.2 & 15.6 & 16.9 & & 14.7 & 8.4 & \\
\hline $\mathrm{PV}$ of public sector debt-to-revenue and grants ratio (in percent) & & & 240.0 & & & 232.7 & 251.0 & 251.6 & 236.9 & 229.9 & 223.0 & & 186.7 & 110.0 & \\
\hline $\mathrm{PV}$ of public sector debt-to-revenue ratio (in percent) & $\ldots$ & $\ldots$ & 256.7 & & & 268.0 & 271.5 & 258.9 & 243.6 & 236.4 & 229.2 & & 191.9 & 113.1 & \\
\hline $\mathrm{o} / \mathrm{w}$ external $3 /$ & $\ldots$ & $\ldots$ & 138.0 & & & 135.5 & 135.7 & 129.6 & 121.9 & 118.5 & 115.1 & & 96.1 & 56.6 & \\
\hline Debt service-to-revenue and grants ratio (in percent) 4/ & 25.6 & 23.8 & 50.1 & & & 46.3 & 32.6 & 40.4 & 35.5 & 44.0 & 42.8 & & 40.4 & 25.3 & \\
\hline Debt service-to-revenue ratio (in percent) $4 /$ & 25.7 & 24.4 & 53.6 & & & 53.4 & 35.2 & 41.6 & 36.5 & 45.2 & 44.0 & & 41.5 & 26.0 & \\
\hline Primary deficit that stabilizes the debt-to-GDP ratio & -3.4 & -2.3 & -6.9 & & & -1.4 & 0.0 & -0.2 & -0.6 & -0.6 & -0.6 & & -0.5 & -0.5 & \\
\hline \multicolumn{16}{|l|}{ Key macroeconomic and fiscal assumptions } \\
\hline Real GDP growth (in percent) & 1.5 & 0.7 & -3.6 & 1.2 & 3.1 & 0.5 & 4.1 & 3.5 & 3.0 & 3.0 & 3.0 & 2.9 & 3.0 & 3.0 & 3.0 \\
\hline Average nominal interest rate on forex debt (in percent) & 5.5 & 4.3 & 4.4 & 4.2 & 1.0 & 5.3 & 5.3 & 5.3 & 5.3 & 5.3 & 5.3 & 5.3 & 5.3 & 5.3 & 5.3 \\
\hline Average real interest rate on domestic debt (in percent) & 4.1 & 5.5 & 4.2 & 4.5 & 1.6 & 2.6 & 4.5 & 4.3 & 4.3 & 4.4 & 4.5 & 4.1 & 4.9 & 4.8 & 4.8 \\
\hline Real exchange rate depreciation (in percent, + indicates depreciation) & 1.3 & 1.0 & -0.5 & 0.2 & 1.3 & -2.0 & $\ldots$ & $\ldots$ & ... & $\ldots$ & $\ldots$ & $\ldots$ & & $\ldots$ & $\ldots$ \\
\hline Inflation rate (GDP deflator, in percent) & 1.6 & 1.1 & 1.4 & 2.2 & 1.3 & 3.0 & 1.6 & 2.0 & 2.1 & 2.1 & 2.1 & 2.2 & 2.2 & 2.3 & 2.2 \\
\hline Growth of real primary spending (deflated by GDP deflator, in percent) & -0.1 & 0.1 & 0.1 & 0.0 & 0.1 & 0.1 & 0.0 & -0.1 & 0.0 & 0.0 & 0.0 & 0.0 & 0.0 & 0.0 & 0.0 \\
\hline Grant element of new external borrowing (in percent) & $\ldots$ & $\ldots$ & $\ldots$ & 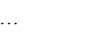 & & -6.4 & -2.5 & -6.4 & -6.4 & -6.8 & -7.0 & -5.9 & -6.8 & -6.4 & \\
\hline
\end{tabular}

Sources: Country authorities; and staff estimates and projections.
/ The analysis covers the public sector guaranteed and non-guaranteed debt and gross debt is used.

$2 /$ Gross financing need is defined as the primary deficit plus debt service plus the stock of short-term debt at the end of the last period.

$3 /$ Revenues excluding grants.

4/ Debt service is defined as the sum of interest and amortization of medium and long-term debt.

$5 /$ Historical averages and standard deviations are generally derived over the past 10 years, subject to data availability. 
Table 2a.St. Lucia: Sensitivity Analysis for Key Indicators of Public Debt 2010-2030

\begin{tabular}{ll}
\hline & \\
\hline Baseline & PV of Debt-to-GDP Ratio
\end{tabular}

Projections

\begin{tabular}{llllllll}
\hline 2010 & 2011 & 2012 & 2013 & 2014 & 2015 & 2020 & 2030 \\
\hline
\end{tabular}

Baseline

A. Alternative scenarios

A1. Real GDP growth and primary balance are at historical averages

A2. Primary balance is unchanged from 2010

A3. Permanently lower GDP growth $1 /$

\section{B. Bound tests}

B1. Real GDP growth is at historical average minus one standard deviations in 2011-2012

B2. Primary balance is at historical average minus one standard deviations in 2011-2012

B3. Combination of B1-B2 using one half standard deviation shocks

B4. One-time 30 percent real depreciation in 2011

B5. 10 percent of GDP increase in other debt-creating flows in 2011

PV of Debt-to-Revenue Ratio 2/

Baseline

A. Alternative scenarios

A1. Real GDP growth and primary balance are at historical averages

A2. Primary balance is unchanged from 2010

A3. Permanently lower GDP growth $1 /$

\section{B. Bound tests}

B1. Real GDP growth is at historical average minus one standard deviations in 2011-2012

B2. Primary balance is at historical average minus one standard deviations in 2011-2012

$\mathrm{B}$. Combination of B1-B2 using one half standard deviation shocks

B4. One-time 30 percent real depreciation in 2011

B5. 10 percent of GDP increase in other debt-creating flows in 2011

Debt Service-to-Revenue Ratio 2/

Baseline

A. Alternative scenarios

A1. Real GDP growth and primary balance are at historical averages

A2. Primary balance is unchanged from 2010

A3. Permanently lower GDP growth 1/

\section{B. Bound tests}

B1. Real GDP growth is at historical average minus one standard deviations in 2011-2012

B3. Combination of B1-B2 using one half standard deviation shocks

B5. 10 percent of GDP increase in other debt-creating flows in 2011
B2. Primary balance is at historical average minus one standard deviations in 2011-2012

B4. One-time 30 percent real depreciation in 2011

$\begin{array}{llllllll}78 & 80 & 79 & 77 & 74 & 72 & 60 & 36\end{array}$

$\begin{array}{llllllll}78 & 82 & 86 & 89 & 93 & 97 & 118 & 169\end{array}$

$\begin{array}{llllllll}78 & 81 & 83 & 87 & 90 & 93 & 110 & 143\end{array}$

$\begin{array}{rrrrrrrr}78 & 81 & 81 & 80 & 79 & 78 & 77 & 92\end{array}$

$\begin{array}{rrrrrrrr}78 & 87 & 94 & 96 & 97 & 99 & 106 & 121 \\ 78 & 82 & 87 & 84 & 82 & 80 & 68 & 44 \\ 78 & 84 & 91 & 91 & 92 & 92 & 94 & 98 \\ 78 & 98 & 97 & 95 & 93 & 91 & 83 & 69 \\ 78 & 90 & 89 & 87 & 85 & 83 & 71 & 47\end{array}$

$\begin{array}{llllllll}233 & 251 & 252 & 237 & 230 & 223 & 187 & 110\end{array}$

$\begin{array}{llllllll}233 & 256 & 272 & 275 & 287 & 299 & 363 & 516 \\ 233 & 253 & 266 & 268 & 278 & 288 & 338 & 443\end{array}$

$\begin{array}{llllllll}233 & 253 & 257 & 246 & 243 & 241 & 238 & 283\end{array}$

$\begin{array}{llllllll}233 & 271 & 300 & 295 & 300 & 305 & 327 & 373 \\ 233 & 258 & 276 & 261 & 254 & 247 & 211 & 136 \\ 233 & 264 & 289 & 281 & 283 & 284 & 290 & 301 \\ 233 & 306 & 308 & 292 & 286 & 281 & 255 & 215 \\ 233 & 284 & 285 & 269 & 262 & 255 & 220 & 145\end{array}$

$\begin{array}{llllllll}46 & 33 & 40 & 35 & 44 & 43 & 40 & 25\end{array}$

$\begin{array}{llllllll}46 & 33 & 42 & 38 & 49 & 51 & 66 & 93 \\ 46 & 33 & 41 & 36 & 46 & 47 & 60 & 78\end{array}$

$\begin{array}{llllllll}46 & 33 & 41 & 36 & 45 & 45 & 47 & 51\end{array}$

$\begin{array}{llllllll}46 & 34 & 46 & 41 & 52 & 52 & 61 & 68 \\ 46 & 33 & 41 & 37 & 47 & 46 & 45 & 30 \\ 46 & 34 & 44 & 39 & 50 & 50 & 56 & 57 \\ 46 & 36 & 48 & 42 & 53 & 53 & 58 & 54 \\ 46 & 33 & 42 & 38 & 49 & 48 & 47 & 32\end{array}$

Sources: Country authorities; and staff estimates and projections.

1/ Assumes that real GDP growth is at baseline minus one standard deviation divided by the square root of the length of the projection period.

2/ Revenues are defined inclusive of grants. 
Table 3a.: External Debt Sustainability Framework, Baseline Scenario, 2007-2030 1/

(In percent of GDP, unless otherwise indicated)

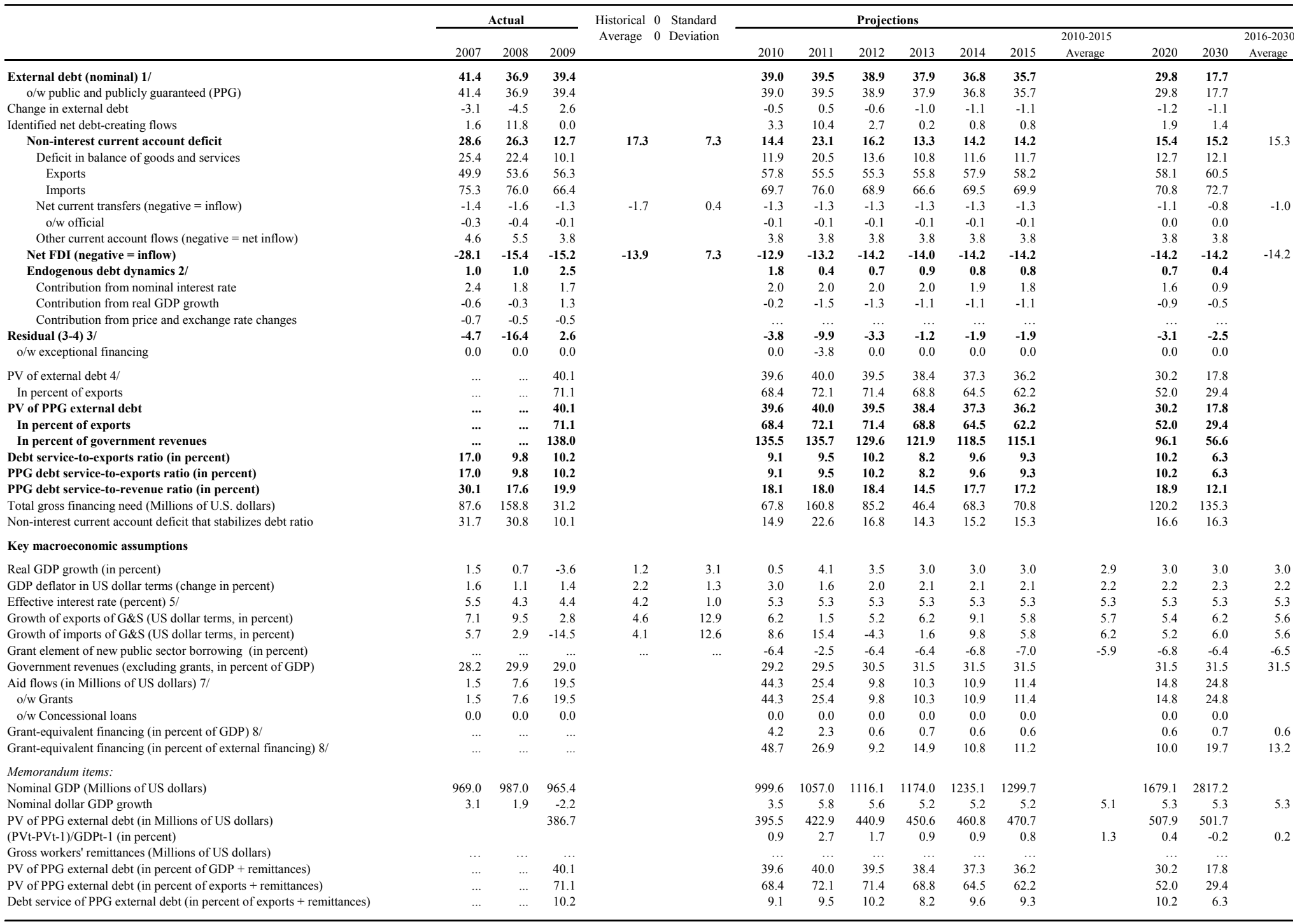

Sources: Country authorities; and staff estimates and projections.

1/ Includes public sector guaranteed and non-guaranteed external debs.

/ Derived as $[\mathrm{r}-\mathrm{g}-\rho(1+\mathrm{g})] /(1+\mathrm{g}+\rho+g \rho)$ times previous period debt ratio, with $\mathrm{r}=$ nominal interest rate; $\mathrm{g}=$ real GDP growth rate, and $\rho=$ growth rate of GDP deflator in US. dollar terms.

3/ Includes exceptional financing (i.e., identified financing for Hurricane

Assumes that PV of private sector debt is equivalent to its face value.

6/ Historical averages pastents 10 years, subject to data avallability.

Defined as grants, concessional loans, and debt relief.

8/ Grant-equivalent financing includes grants provided directly to the government and through new borrowing (difference between the face value and the PV of new debt) 
Table 3b.St. Lucia: Sensitivity Analysis for Key Indicators of Public and Publicly Guaranteed External Debt, 2010-2030 (In percent)

\begin{tabular}{|c|c|c|c|c|c|c|c|c|}
\hline & \multicolumn{8}{|c|}{ Projections } \\
\hline & 2010 & 2011 & 2012 & 2013 & 2014 & 2015 & 2020 & 2030 \\
\hline \multicolumn{9}{|c|}{ PV of debt-to GDP ratio } \\
\hline Baseline & 40 & 40 & 40 & 38 & 37 & 36 & 30 & 18 \\
\hline \multicolumn{9}{|l|}{ A. Alternative Scenarios } \\
\hline A1. Key variables at their historical averages in 2010-2030 1/ & 40 & 34 & 36 & 40 & 44 & 47 & 52 & 47 \\
\hline A2. New public sector loans on less favorable terms in 2010-2030 2 & 40 & 41 & 40 & 40 & 39 & 38 & 31 & 20 \\
\hline \multicolumn{9}{|l|}{ B. Bound Tests } \\
\hline B1. Real GDP growth at historical average minus one standard deviation in 2011-2012 & 40 & 42 & 44 & 43 & 42 & 41 & 34 & 20 \\
\hline B2. Export value growth at historical average minus one standard deviation in 2011-2012 3/ & 40 & 46 & 58 & 56 & 53 & 50 & 35 & 18 \\
\hline B3. US dollar GDP deflator at historical average minus one standard deviation in 2011-2012 & 40 & 40 & 40 & 39 & 38 & 37 & 31 & 18 \\
\hline B4. Net non-debt creating flows at historical average minus one standard deviation in 2011-2012 4/ & 40 & 47 & 55 & 53 & 50 & 47 & 34 & 18 \\
\hline B5. Combination of B1-B4 using one-half standard deviation shocks & 40 & 47 & 61 & 59 & 56 & 53 & 38 & 20 \\
\hline B6. One-time 30 percent nominal depreciation relative to the baseline in 20115 / & 40 & 57 & 56 & 54 & 53 & 51 & 43 & 25 \\
\hline
\end{tabular}

\section{PV of debt-to-exports ratio}

\section{Baseline}

A. Alternative Scenarios

A1. Key variables at their historical averages in 2010-2030 1/

A2. New public sector loans on less favorable terms in 2010-2030 2

\section{B. Bound Tests}

B1. Real GDP growth at historical average minus one standard deviation in 2011-2012

B2. Export value growth at historical average minus one standard deviation in 2011-2012 3/

B3. US dollar GDP deflator at historical average minus one standard deviation in 2011-2012

B4. Net non-debt creating flows at historical average minus one standard deviation in 2011-2012 4/

B5. Combination of B1-B4 using one-half standard deviation shocks

B6. One-time 30 percent nominal depreciation relative to the baseline in 20115 /

$\begin{array}{rrrrrrrr}68 & 72 & 71 & 69 & 64 & 62 & \mathbf{5 2} & 29 \\ & & & & & & & \\ 68 & 61 & 64 & 72 & 76 & 81 & \mathbf{8 9} & 78 \\ 68 & 73 & 73 & 71 & 67 & 65 & \mathbf{5 3} & 34 \\ & & & & & & & \\ & & & & & & & \\ 68 & 72 & 71 & 69 & 64 & 62 & \mathbf{5 2} & 29 \\ 68 & 91 & 132 & 127 & 116 & 109 & \mathbf{7 7} & 38 \\ 68 & 72 & 71 & 69 & 64 & 62 & \mathbf{5 2} & 29 \\ 68 & 85 & 99 & 94 & 86 & 81 & \mathbf{5 9} & 30 \\ 68 & 85 & 111 & 107 & 98 & 92 & \mathbf{6 6} & 33 \\ 68 & 72 & 71 & 69 & 64 & 62 & \mathbf{5 2} & 29\end{array}$

PV of debt-to-revenue ratio

Baseline

\section{A. Alternative Scenarios}

A1. Key variables at their historical averages in 2010-2030 1/

A2. New public sector loans on less favorable terms in 2010-2030 2

$\begin{array}{rrrrrrrr}136 & 115 & 117 & 128 & 140 & 150 & \mathbf{1 6 5} & 150 \\ 136 & 138 & 133 & 126 & 124 & 120 & \mathbf{9 8} & 64\end{array}$

B. Bound Tests

B1. Real GDP growth at historical average minus one standard deviation in 2011-2012

B2. Export value growth at historical average minus one standard deviation in 2011-2012 3/

B3. US dollar GDP deflator at historical average minus one standard deviation in 2011-2012

B4. Net non-debt creating flows at historical average minus one standard deviation in 2011-2012 4/

B5. Combination of B1-B4 using one-half standard deviation shocks

B6. One-time 30 percent nominal depreciation relative to the baseline in 2011 5/

$\begin{array}{rrrrrrrr}136 & 144 & 145 & 137 & 133 & 129 & \mathbf{1 0 8} & 63 \\ 136 & 155 & 189 & 177 & 168 & 159 & \mathbf{1 1 2} & 57 \\ 136 & 137 & 132 & 124 & 121 & 117 & \mathbf{9 8} & 58 \\ 136 & 160 & 179 & 167 & 159 & 151 & \mathbf{1 0 8} & 57 \\ 136 & 161 & 199 & 187 & 177 & 168 & \mathbf{1 2 0} & 62 \\ 136 & 193 & 184 & 173 & 168 & 163 & \mathbf{1 3 6} & 80\end{array}$


Table 3b.St. Lucia: Sensitivity Analysis for Key Indicators of Public and Publicly Guaranteed External Debt, $2010-2030$ (continued) (In percent)

\begin{tabular}{|c|c|c|c|c|c|c|c|c|}
\hline & \multicolumn{8}{|c|}{ Projections } \\
\hline & 2010 & 2011 & 2012 & 2013 & 2014 & 2015 & 2020 & 2030 \\
\hline \multicolumn{9}{|c|}{ Debt service-to-exports ratio } \\
\hline Baseline & 9 & 10 & 10 & 8 & 10 & 9 & 10 & 6 \\
\hline \multicolumn{9}{|l|}{ A. Alternative Scenarios } \\
\hline A1. Key variables at their historical averages in 2010-2030 1/ & 9 & 9 & 10 & 7 & 9 & 10 & 16 & 16 \\
\hline A2. New public sector loans on less favorable terms in 2010-2030 2 & 9 & 9 & 10 & 8 & 10 & 10 & 11 & 7 \\
\hline \multicolumn{9}{|l|}{ B. Bound Tests } \\
\hline B1. Real GDP growth at historical average minus one standard deviation in 2011-2012 & 9 & 9 & 10 & 8 & 9 & 9 & 10 & 6 \\
\hline B2. Export value growth at historical average minus one standard deviation in 2011-2012 3/ & 9 & 10 & 13 & 13 & 17 & 17 & 17 & 8 \\
\hline B3. US dollar GDP deflator at historical average minus one standard deviation in 2011-2012 & 9 & 9 & 10 & 8 & 9 & 9 & 10 & 6 \\
\hline B4. Net non-debt creating flows at historical average minus one standard deviation in 2011-2012 4/ & 9 & 9 & 10 & 10 & 13 & 13 & 13 & 6 \\
\hline B5. Combination of B1-B4 using one-half standard deviation shocks & 9 & 9 & 11 & 11 & 15 & 14 & 14 & 7 \\
\hline B6. One-time 30 percent nominal depreciation relative to the baseline in 20115 / & 9 & 9 & 10 & 8 & 9 & 9 & 10 & 6 \\
\hline
\end{tabular}

Debt service-to-revenue ratio

\section{Baseline}

A. Alternative Scenarios

A1. Key variables at their historical averages in 2010-2030 1/

A2. New public sector loans on less favorable terms in 2010-2030 2

\section{B. Bound Tests}

B1. Real GDP growth at historical average minus one standard deviation in 2011-2012

B2. Export value growth at historical average minus one standard deviation in 2011-2012 3/

B3. US dollar GDP deflator at historical average minus one standard deviation in 2011-2012

B4. Net non-debt creating flows at historical average minus one standard deviation in 2011-2012 4/

B5. Combination of B1-B4 using one-half standard deviation shocks

B6. One-time 30 percent nominal depreciation relative to the baseline in $20115 /$

Memorandum item

Grant element assumed on residual financing (i.e., financing required above baseline) 6 /

Sources: Country authorities; and staff estimates and projections.

1/ Variables include real GDP growth, growth of GDP deflator (in U.S. dollar terms), non-interest current account in percent of GDP, and non-debt creating flows.

2/ Assumes that the interest rate on new borrowing is by 2 percentage points higher than in the baseline., while grace and maturity periods are the same as in the baseline.

3/ Exports values are assumed to remain permanently at the lower level, but the current account as a share of GDP is assumed to return to its baseline level after the shock (implicitly assuming an offsetting adjustment in import levels).

4/ Includes official and private transfers and FDI.

5/ Depreciation is defined as percentage decline in dollar/local currency rate, such that it never exceeds 100 percent.

6/ Applies to all stress scenarios except for A2 (less favorable financing) in which the terms on all new financing are as specified in footnote 2. 
Press Release No. 11/6

International Monetary Fund

FOR IMMEDIATE RELEASE

Washington, D.C. 20431 USA

January 12, 2011

\section{IMF Executive Board Approves US\$8.2 Million in Emergency Assistance for St. Lucia}

The Executive Board of the International Monetary Fund (IMF) today approved a combined SDR 5.36 million (about US\$8.19 million) in emergency assistance for St. Lucia to cope with the economic consequences of Hurricane Tomas. The financial assistance consists of an SDR 3.83 million (about US\$5.85 million) disbursement under the IMF's Rapid Credit Facility (RCF), and SDR 1.53 million ( about US\$2.34 million) under the Fund's Emergency Natural Disaster Assistance (ENDA).

Hurricane Tomas struck St. Lucia on October 30, 2010 causing loss of life and significant damage to the nation's road network, water supply, and agriculture sector. The latest but still preliminary estimates suggest that total damages amount to US\$336 million, or about 34 percent of GDP.

Following the Executive Board discussion of St. Lucia, Mr. Murilo Portugal, Deputy Managing Director and Acting Chair, issued the following statement:

"St. Lucia has been severely affected by Hurricane Tomas, which caused loss of life and significant damages to infrastructure and agriculture. The reconstruction efforts are expected to have a significant fiscal and balance of payment impact, mainly in 2011, posing difficult policy challenges. It will be important to maintain macroeconomic stability and debt sustainability during the rehabilitation.

"Despite a temporary deterioration in the fiscal position, the authorities remain committed to the medium-term debt target. They intend to keep commercial borrowing to a minimum by limiting the financing of the rehabilitation and reconstruction efforts to available grants and external concessional financing. Key consolidation measures include introducing a market valuation-based property tax in early 2011 and a value-added tax by April 2012, and controlling the civil service wage bill over the medium term. A formal public sector investment program will also be initiated, to increase the efficiency of spending and underpinned by a medium-term expenditure framework. 
"The authorities continue to make progress on other structural reforms. Efforts are ongoing to enhance the investment climate conducive to private sector growth, and to strengthen the oversight and resilience of the financial sector, including through the establishment of the Single Regulatory Unit.

“The authorities' prudent management of the reconstruction efforts, together with the continued sound policies to enhance the fiscal position and address structural issues, will help St. Lucia maintain fiscal sustainability and achieve the medium-term debt target," Mr. Portugal said.

\section{Rapid Credit Facility}

\section{ANNEX}

The RCF provides rapid concessional financial assistance with limited conditionality to lowincome countries facing an urgent balance of payments need, such as those caused by natural disasters.

The RCF streamlines the Fund's emergency assistance, provides significantly higher levels of concessionality, can be used flexibly in a wide range of circumstances, and places greater emphasis on the country's poverty reduction and growth objectives. Financing under the RCF carries a zero interest rate, has a grace period of 5 $\frac{1}{2}$ years, and a maturity of 10 years.

\section{Emergency Natural Disaster Assistance}

Since 1962, the IMF has provided emergency assistance to member countries afflicted by natural disasters such as floods, earthquakes, hurricanes, or droughts. This assistance, provided under the Emergency Natural Disaster Assistance (ENDA), meets immediate foreign exchange financing needs arising from, e.g., shortfalls in export earnings and/or increased imports, and avoiding a serious depletion of external reserves. Emergency assistance loans are usually quick-disbursing and do not involve adherence to performance criteria. 


\section{Statement by Stephen O'Sullivan, Executive Director for St. Lucia January 12, 2011}

The authorities wish to thank the staff for their swift response in the wake of the hurricane which struck the country on 30/31 October 2010. A staff team was in place in St. Lucia just three weeks later for discussions on how best to support the authorities' efforts to cope with the immediate aftermath of the disaster. These discussions led to the formulation of a request for a disbursement under the Rapid Credit Facility and the Emergency Natural Disaster Assistance. The authorities would be grateful for Board approval of that request. As well as helping directly to meet St. Lucia's immediate balance of payments financing needs, Board approval would facilitate the mobilization of resources from other institutions.

\section{Impact of the Hurricane}

Hurricane Tomas was an event of historic proportions for St. Lucia. The level of rainfall recorded in a 24 hour period was double that recorded during tropical storm Debbie in 1994 and has been assessed as a 1 in 180 year event. The entire population was impacted by the reduction in the water supply to just 1 percent of the pre-hurricane supply. Sanitation was a major issue as a large portion of the population was without access to safe water. There was extensive damage to roads, forestry and to the banana crop. Preliminary estimates of the damage to the banana industry assessed the impact at 80 to 90 percent losses. The infrastructure of the agricultural sector received major damage and will require much reinvestment. Large landslides occurred, affecting transport infrastructure. Hotels were badly affected and tourism was severely disrupted. Reconstruction work will be needed in housing, roads, water supply, river courses, forestry, the banana industry, the tourism sector and economic infrastructure generally. The staff report puts the overall impact of the hurricane at 34 percent of GDP.

\section{Economic Context}

The focus of the authorities has for some time been on managing the transition from an agricultural economy dominated by a single product to a more modern services-based economy. Prior to the downturn in the global economy, St. Lucia had been making appreciable strides towards this goal. GDP growth, supported by significant volumes of foreign direct investment, was running at around 4 percent. However, gross public sector debt was uncomfortably high at around 67 percent of GDP and debt service costs were absorbing almost 40 percent of current revenues. This level of indebtedness reflected in part the commitment of the authorities to invest heavily in infrastructural development. Evidence that this was paying off was to be seen in the marked increase in tourism capacity and the growth in tourism earnings. However, as the staff report says, it will take considerable efforts and resources to put the economy back on its pre-hurricane growth path. 
The hurricane of August 2007 and the earthquake later that year combined with the subsequent downturn in the world economy to disrupt progress. These events led to a sharp fall in output in 2009 which, together with the necessary countervailing policy measures introduced by the authorities, resulted in a weakening of the government accounts and an increase in the burden of public debt of around 12 percentage points of GDP.

\section{Recent Economic Developments}

Prior to the hurricane, St. Lucia was on track to outperform expectations at the time of the Article IV discussions last March. As indicated in the staff report, the pre-hurricane expectation was for GDP growth of 1.7 percent, some 0.6 percentage points above previous forecasts. The primary deficit, at 0.6 percent of GDP, would have been just one-fifth of that envisaged in March 2010 and the cash deficit, at 4.3 percent, would have been three percentage points lower. The principal driver of this improvement was the very strong performance of the tourism sector which was significantly outstripping expectations, validating the earlier commitment of the authorities to invest in necessary infrastructural upgrades.

\section{Economic Prospects}

The outlook for 2011 is for a return to a growth rate of around 4 percent. This exceeds prehurricane expectations, reflecting a stimulus effect from post-hurricane activity and a recovery in tourist arrivals. Looking further ahead, however, debt dynamics have altered in that the primary surplus required to achieve the regional objective of a 60 percent debt level by 2020 has increased from around 1.6 percent to 2.9 percent.

\section{Policy Issues}

The policy issues mentioned in the course of the March 2010 Article IV discussions remain on the table. The central issue is still the need to assure debt sustainability within the context of economic development and poverty reduction. Financial sector stability remains on the agenda and the warning note sounded by staff on the occasion of the Article IV review in relation to the incidence of NPLs is still relevant.

The full suite of policy issues will be taken up in the context of the Article IV review which will take place in the coming months. In the course of those discussions, the authorities will return to the issues touched on in the Prime Minister's Letter of Intent which accompanies the current request, including the recent progress on legislation to introduce a market-based property tax and their commitment to introduce a VAT next year. They will also set out their intentions in relation to the control of public sector wage costs and their plans for a mediumterm expenditure framework. 


\section{Conclusion}

The authorities welcome staff's acknowledgement of their solid track record of sound fiscal policies and of close collaboration with the Fund. In return, they wish to express their appreciation for the support shown by the Executive Board of their efforts. They would be grateful for Board approval of the request for a purchase and disbursement under ENDA and the RCF amounting to 35 percent of quota and they look forward to re-engaging more fully on the full set of policy issues during the forthcoming Article IV consultations. 